\title{
Joint Trajectory and Precoding Optimization for UAV-Assisted NOMA Networks
}

\author{
Nan Zhao, Senior Member, IEEE, Xiaowei Pang, Zan Li, Senior Member, IEEE, Yunfei Chen, Senior \\ Member, IEEE, Feng Li, Zhiguo Ding, Senior Member, IEEE, and Mohamed-Slim Alouini, Fellow, IEEE
}

\begin{abstract}
The explosive data traffic and connections in 5G networks require the use of non-orthogonal multiple access (NOMA) to accommodate more users. Unmanned aerial vehicle (UAV) can be exploited with NOMA to improve the situation further. In this paper, we propose a UAV-assisted NOMA network, in which the UAV and base station (BS) cooperate with each other to serve ground users simultaneously. The sum rate is maximized by jointly optimizing the UAV trajectory and the NOMA precoding. To solve the optimization, we decompose it into two steps. First, the sum rate of the UAV-served users is maximized via alternate user scheduling and UAV trajectory, with its interference to the BS-served users below a threshold. Then, the optimal NOMA precoding vectors are obtained using two schemes with different constraints. The first scheme intends to cancel the interference from the BS to the UAV-served user, while the second one restricts the interference to a given threshold. In both schemes, non-convex optimization problems are converted into tractable ones. An iterative algorithm is designed. Numerical results are provided to evaluate the effectiveness of the proposed algorithms for the hybrid NOMA and UAV network.
\end{abstract}

Index Terms-Interference avoidance, non-orthogonal multiple access, precoding, trajectory optimization, unmanned aerial vehicle.

Manuscript received May 18, 2018; revised September 10, 2018, October 31, 2018 and December 22, 2018; accepted January 21, 2019. The work of N. Zhao was supported by the National Natural Science Foundation of China (NSFC) under Grant 61871065, the open research fund of State Key Laboratory of Integrated Services Networks under Grant ISN19-02, the Fundamental Research Funds for the Central Universities under DUT17JC43, and the Xinghai Scholars Program. The work of Z. Li was supported by the Key project of National Natural Science Foundation of China (NSFC) under Grant 61631015 and the Fundamental Research Funds for the Central Universities under 7215433803. The work of Z. Ding was supported by the UK EPSRC under grant number EP/N005597/1, NSFC under grant number 61728101 and H2020-MSCA-RISE-2015 under grant number 690750. Part of this work has been published in preliminary form in the Proceedings of WCSP 2018 [1]. The associate editor coordinating the review of this paper and approving it for publication was W. Chen. (Corresponding author: Feng Li.)

N. Zhao and X. Pang are with the School of Information and Communication Engineering, Dalian University of Technology, Dalian 116024, P. R. China, and also with the State Key Laboratory of Integrated Services Networks, Xidian University, Xi'an, 710071, P. R. China (email: zhaonan@dlut.edu.cn, xiaoweipang00@mail.dlut.edu.cn).

$\mathrm{Z}$. Li is with the State Key Laboratory of Integrated Services Networks, Xidian University, Xi'an 710071, P. R. China (Email: zanli@ xidian.edu.cn).

Y. Chen is with the School of Engineering, University of Warwick, Coventry CV4 7AL, U.K. (e-mail: Yunfei.Chen@warwick.ac.uk).

F. Li is with the College of Information Engineering, Zhejiang University of Technology, Hangzhou, 310023, P. R. China (fengli2002@yeah.net).

Z. Ding is with the School of Electrical and Electronic Engineering, The University of Manchester, Manchester, M13 9PL, U.K. (e-mail: zhiguo.ding@manchester.ac.uk).

Mohamed-Slim Alouini is with the Computer, Electrical, and Mathematical Science and Engineering (CEMSE) Division, King Abdullah University of Science and Technology (KAUST), Thuwal, Makkah Province, Saudi Arabia. (e-mail: slim.alouini@kaust.edu.sa).

\section{INTRODUCTION}

The high spectrum efficiency requirement and massive connections become the primary challenges for $5 \mathrm{G}$ networks [2]. As one of the crucial techniques for $5 \mathrm{G}$, non-orthogonal multiple access (NOMA) can not only significantly improve the spectrum efficiency but also allow more users or devices to access the network [3]-[7]. Different from the conventional orthogonal multiple access (OMA), NOMA is built upon the idea that multiple users can simultaneously share one resource block, such as a subcarrier or a spreading code [3]. These users are multiplexed in the power domain, and successive interference cancellation (SIC) is required at the receiver for demodulation [4]. The key inspiration of SIC is to leverage the difference among signal strengths to decode users with signals of higher power from others successively.

Due to its superior performance, NOMA has attracted great research interest from both academia and industry [8]-[21]. In [8], the key features and practical aspects of the OMA and NOMA techniques were discussed and compared. Chen et al. presented rigorous mathematical proofs in [9] to demonstrate that NOMA can always outperform the conventional OMA, when both use optimal resource allocation policies. In [10], Yang et al. proposed a novel dynamic power allocation scheme, offering more flexibility for the tradeoff between user fairness and system throughput. Since users in a single group sharing one resource block can generate high SIC complexity and decoding delay, Ding et al. studied the impact of user paring on the performance of NOMA in [11]. Hanif $e t$ al. proposed to effectively solve the sum rate maximization problem of a multiple-input-single-output (MISO) NOMA system in [12], by optimizing the weighted precoding vectors. With regard to multiple-input-multiple-output (MIMO) systems, Ding et al. devised a new form of precoding and decoding matrices for NOMA downlink in [13]. For massive MIMO, Lin et al. demonstrated a new prospective of nonorthogonal angle division multiple access in [14]. In [15], a novel secondary NOMA relay assisted spectrum sharing scheme was proposed, which can save significant transmit power when the primary receiver is far from its transmitter. The optimal power allocation and scheduling for NOMA relayassisted network were jointly studied in [16]. In [17], Zhao et al. proposed a novel NOMA multicast scheme through pushing and multicasting the caching contents together to enhance the spectrum efficiency. In addition, the NOMA-based heterogeneous networks have been extensively researched in [18]-[21].

Although NOMA can achieve promising performance, there 
still exist challenges, especially when it is used to provide massive connections, such as internet of things for 5G. Firstly, the quality of service (QoS) of edge users needs to be further improved. In addition, more users should be served in a single subcarrier. This may increase the load and delay of SIC. For these reasons, unmanned aerial vehicles (UAVs) can be exploited as a potential method to assist NOMA to achieve better performance. Due to the flexibility and mobility, UAVs have drawn increasing attention [22], which can be applied to many wireless scenarios including ubiquitous coverage [23], relaying [24], [25], as well as information dissemination and data collection [22]. Recently, plenty of excellent research on UAV communications has been conducted. For example, in [26], $\mathrm{Li}$ and Cai leveraged the UAV as a floating relay in heterogeneous cellular networks to achieve dynamic and adaptive coverage. Zhao et al. proposed a blind beam tracking method for UAV-satellite communication systems in [27] with hybrid massive antennas. Owing to a better air-ground channel, UAVs can be widely adopted as mobile base stations (BSs) in wireless networks [28]-[34]. Some fundamental works have been done by $\mathrm{Wu}$ et al., in which the single-UAV enabled networks and multi-UAV enabled networks were studied in [28], [29] respectively, to maximize the minimum throughput of all ground users. In [30], a novel hybrid network was proposed to use the UAV to help offload data traffic from the BS, where the UAV trajectory was jointly optimized with the bandwidth allocation and user partitioning. Cheng et al. in [31] employed the UAV at the edge of three adjacent cells to offload traffic for BSs by optimizing the UAV trajectory. In [32], UAV-assisted secure transmission via caching was proposed by Zhao et al., to alleviate backhaul pressure and to guarantee security. To further save energy and prolong the batteries, energy-efficient UAV communications were studied by Zeng and Yang et al. in [33], [34].

Although NOMA and UAV have been extensively studied in the previous works, to the best of our knowledge, few works have combined these two promising aspects to overcome their respective shortcomings [1]. In this paper, we study the sum rate maximization in a UAV-assisted NOMA network by jointly optimizing UAV trajectory and NOMA precoding at BS. The main contributions of this paper can be summarized as follows.

- In this paper, we propose a hybrid UAV-assisted network, where time division multiple access (TDMA) is adopted for UAV transmission while NOMA is applied to the ground BS with multi-antennas. Aiming to maximize the sum rate of all users, the joint optimization problem of user scheduling, UAV trajectory and precoding at BS is complicated and combinatorial, which is decomposed into two parts to solve.

- First, the UAV optimization problem is formulated to maximize the throughput of UAV-served users, with a limitation on the interference to BS-served users. For this problem, an iterative algorithm utilizing block coordinate descent is proposed to alternately optimizing the user scheduling and UAV trajectory, which can converge quickly with an initial circular trajectory.

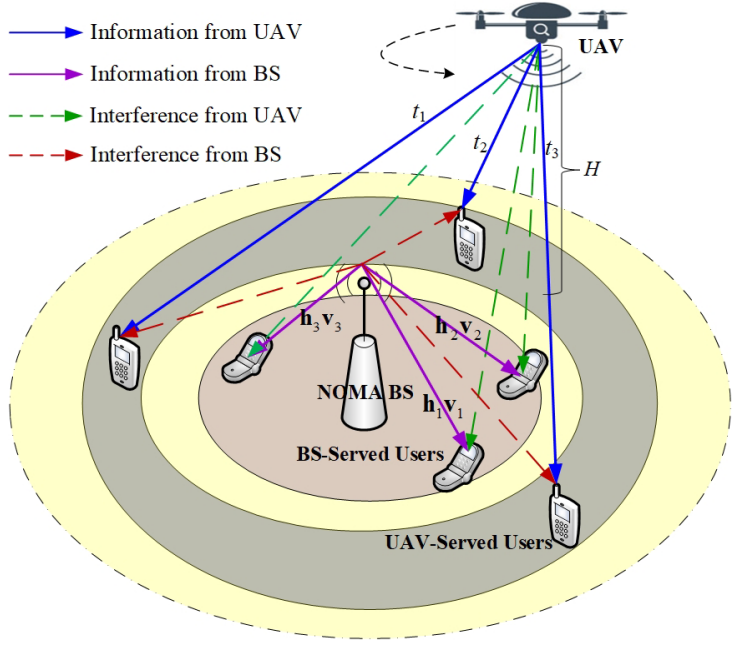

Fig. 1. Demonstration of the UAV-Assisted NOMA Network.

- Based on the optimal scheduling and trajectory of UAV, we design two precoding schemes to maximize the sum rate of BS-served users. Specifically, the first scheme intends to make the interference from the BS to the UAVserved user zero-forced, while the second one restricts the interference by a threshold. For both schemes, nonconvex problems are transformed into convex ones, which can be solved by an iterative algorithm.

The rest of this paper is organized as follows. In Section II, the system model and problem formulation are presented. The UAV trajectory optimization subproblem is described and solved in Section III. In Section IV, the joint precoding optimization for NOMA BS is studied. In Section V, the simulation results and discussion are demonstrated, followed by conclusions in Section VI.

Notation: A represents a matrix while a denotes a vector. $\|\mathbf{A}\|$ and $\mathbf{A}^{\dagger}$ denote the Frobenius norm and Hermitian transpose of $\mathbf{A}$, respectively. $\|\mathbf{a}\|$ is the Euclidean norm of $\mathbf{a}$ and $\mathbb{C}^{M \times N}$ is the space of $M \times N$ complex matrices. $\mathcal{C N}(\mathbf{a}, \mathbf{A})$ indicates the complex Gaussian distribution with mean a and covariance A. $\operatorname{Re}(c)$ is the real part of a complex number $c$.

\section{SySTEM MOdEL}

\section{A. System Model}

Consider a cellular network with a UAV and a NOMA BS jointly serving the ground users as shown in Fig. 1. It is assumed that the ground users are randomly distributed in the cell, the BS is static at the center, and the UAV flies periodically over the area. Denote the sets of users served by the BS and by the UAV as $k \in \mathcal{K} \triangleq\{1,2, \ldots, K\}$ and $i \in \mathcal{I} \triangleq\{1,2, \ldots, I\}$, respectively. In addition, assume that the UAV as well as all the ground users are equipped with a single antenna while the BS has $M$ antennas.

For simplicity, we assume that the UAV flies at a fixed altitude $H$ above the ground and serves its associated users via 
cyclical TDMA ${ }^{1}$ with a constant cycle duration $\mathcal{T}$, which can be equally divided into $\mathcal{N}$ time slots. The value of $\mathcal{N}$ should be carefully chosen to make sure that the location of UAV is approximately unchanged within each slot, and it also needs to satisfy the constraint of $\mathcal{N} \geq I$ to guarantee the fairness. In addition, $v$ denotes the maximum speed of UAV. Without loss of generality, a 3D Cartesian coordinate is adopted in this paper, and thus, we can define the horizon coordinate of the $m$ th ground user as $\mathbf{L}_{m}=\left[x_{m}, y_{m}\right]^{T} m \in \mathcal{I}$ or $m \in \mathcal{K}$ and the location of the BS as $\mathbf{B}=\left[x_{B}, y_{B}\right]^{T}$, respectively. Define the location of the UAV projected on the ground in the $n$th time slot as $\mathbf{w}[n]=[x[n], y[n]]^{T}, n=1,2, \ldots N$. As a result, the trajectory of UAV should satisfy the following constraints.

$$
\begin{gathered}
\mathbf{w}[1]=\mathbf{w}[\mathcal{N}], \\
\|\mathbf{w}[n+1]-\mathbf{w}[n]\|^{2} \leq(v \mathcal{T} / \mathcal{N})^{2}, n=1, \ldots, \mathcal{N}-1,
\end{gathered}
$$

where (1) indicates that the UAV will return to its initial position at the end of each period $\mathcal{T}$, and the term $v \mathcal{T} / \mathcal{N}$ in (2) denotes the maximum distance it can fly during each time slot.

For convenience, we further define a binary variable $s_{i}[n] \in$ $\{0,1\}$ to denote user scheduling for UAV, where $s_{i}[n]=1$ means that the UAV serves the $i$ th user in the $n$th time slot, while $s_{i}[n]=0$ implies that the $i$ th user is not served by the UAV in the $n$th time slot. In addition, we assume that at most one user can be served by the UAV during each time slot. Then, the following constraints can be derived,

$$
\begin{gathered}
s_{i}[n]=\{0,1\}, \forall i \in \mathcal{I}, \forall n, \\
\sum_{i \in \mathcal{I}} s_{i}[n] \leq 1, \forall n .
\end{gathered}
$$

We assume that the wireless links from the UAV to the ground users are dominated by line-of-sight (LoS) and further suppose that the Doppler effect caused by the mobility of UAV can be perfectly compensated [33]-[36]. Thus, the channel coefficient from the UAV to the user located at $\mathbf{L}_{m}(m \in \mathcal{I}$ or $m \in \mathcal{K}$ ) in the $n$th time slot can be given by

$$
h_{u m}[n]=\sqrt{\frac{\rho_{u}}{H^{2}+\left\|\mathbf{w}[n]-\mathbf{L}_{m}\right\|^{2}}}, n=1, \ldots, \mathcal{N},
$$

where $\rho_{u}$ denotes the reference channel power gain at the distance $d_{0}=1 \mathrm{~m}$ from the UAV. For the channel fading vector from the BS to a ground user, it can be expressed as

$$
\mathbf{h}_{m}=\sqrt{\rho_{b} d_{m}^{-\alpha}} \mathbf{g}_{m} \in \mathbb{C}^{1 \times M}, \forall m \in\{\mathcal{I}, \mathcal{K}\},
$$

where $\rho_{b}$ indicates the channel power gain at the reference distance $d_{0}=1 \mathrm{~m}$ from the BS, $d_{m}=\left\|\mathbf{B}-\mathbf{L}_{m}\right\|$ is the distance from the $m$ th user to the $\mathbf{B S}$, and $\alpha$ is the path loss exponent. In addition, $\mathbf{g}_{m} \sim \mathcal{C N}(\mathbf{0}, \mathbf{I})$ follows Rayleigh fading.

It is assumed that NOMA is employed by the ground BS to transmit data to its associated users, with the transmitted signals precoded by complex vectors. Thus, the $i$ th user in

\footnotetext{
${ }^{1}$ Although there exist research on the UAV networks based on OFDMA [35] and NOMA [36], [37], we adopt TDMA, which can further improve spectrum efficiency, save transmit power and reduce decoding complexity in the scenario of this paper.
}

$\mathcal{I}$ receives its desired signal from UAV when it is served, together with the superposed signals from the BS as interference, which yields

$$
y_{i}[n]=h_{u i}[n] x_{i}+\sum_{k=1}^{K} \mathbf{h}_{i} \mathbf{v}_{k} x_{k}+n_{i}, s_{i}[n]=1, \forall n,
$$

where $\mathbf{v}_{k} \in \mathbb{C}^{M \times 1}$ is the complex precoding vector for the $k$ th user served by the BS, $\left\|\mathbf{v}_{k}\right\|^{2}=P_{k}, k \in \mathcal{K}$. By properly designing $\mathbf{v}_{k}$, the sum rate of BS-served users can be significantly improved and the interference from the BS to the UAV-served user can be constrained as well. The transmitted signal for the $m$ th user with unit power is expressed as $x_{m}$ $(m \in \mathcal{I}$ or $m \in \mathcal{K})$ and $n_{i} \sim \mathcal{C N}\left(0, \sigma^{2}\right)$ represents the additive white Gaussian noise (AWGN) at the $i$ th user.

In each time slot, the $k$ th user in $\mathcal{K}$ receives the superposed signals from the BS along with the interference from the UAV, which can be expressed as

$$
y_{k}[n]=\sum_{j=1}^{K} \mathbf{h}_{k} \mathbf{v}_{j} x_{j}+h_{u k}[n] x_{i}+n_{k}, \forall k \in \mathcal{K}, \forall n .
$$

During this time slot, the $i$ th user is served by the UAV, i.e., $s_{i}[n]=1, \forall i \in \mathcal{I}$.

For the performance of UAV transmission, we adopt the average rate of the $i$ th user served by UAV over the $\mathcal{N}$ time slots of a cycle to illustrate its QoS, which can be given by

$$
R_{u}^{i}=\frac{1}{\mathcal{N}} \sum_{n=1}^{\mathcal{N}} s_{i}[n] \log _{2}\left(1+\frac{\frac{p \rho_{u}}{H^{2}+\left\|\mathbf{w}[n]-\mathbf{L}_{i}\right\|^{2}}}{I_{b}^{i}+\sigma^{2}}\right), \forall i \in \mathcal{I},
$$

where

$$
I_{b}^{i}=\sum_{k \in \mathcal{K}}\left|\mathbf{h}_{i} \mathbf{v}_{k}\right|^{2},
$$

is the interference from the BS to the $i$ th user served by UAV, and $p$ is the UAV transmit power.

According to NOMA, SIC is adopted at each BS-served user based on the signal strengths. It is assumed that the channel strengths of users are in an ascending order with respect to their index numbers, i.e., $\left\|\mathbf{h}_{1}\right\|^{2} \leq\left\|\mathbf{h}_{2}\right\|^{2} \leq \ldots \leq\left\|\mathbf{h}_{k}\right\|^{2} \leq$ $\ldots \leq\left\|\mathbf{h}_{K}\right\|^{2}$. Larger $k$ means that better condition with lower transmit power, and we call it "stronger user". On the contrary, smaller $k$ means that poorer condition with higher transmit power, and we call it "weaker user". In NOMA networks, it is necessary for the $k$ th user to decode and subtract signals of the weaker users with higher transmit power whose index $j<k$ before recovering its own message. In addition, the $k$ th user served by BS also suffers the interference from the UAV, which can be expressed as

$$
I_{u}^{k}=p \rho_{u} /\left(H^{2}+\left\|\mathbf{w}[n]-\mathbf{L}_{k}\right\|^{2}\right), \forall k \in \mathcal{K} .
$$

To perform SIC and decode the desired information of each user successfully, the following condition should be satisfied.

$$
I_{u}^{k} \leq\left|\mathbf{h}_{k} \mathbf{v}_{K}\right|^{2} \leq\left|\mathbf{h}_{k} \mathbf{v}_{K-1}\right|^{2} \leq \cdots \leq\left|\mathbf{h}_{k} \mathbf{v}_{1}\right|^{2}, \forall k \in \mathcal{K} .
$$

Thus, $I_{u}^{k}$ can be deemed as noise, which should be minimized by the optimization of UAV. Then, the achievable rate of the $k$ th user via SIC can be denoted as

$$
R_{b}^{k}=\log _{2}\left(1+\frac{\left|\mathbf{h}_{k} \mathbf{v}_{k}\right|^{2}}{\sum_{j=k+1}^{K}\left|\mathbf{h}_{k} \mathbf{v}_{j}\right|^{2}+I_{u}^{k}+\sigma^{2}}\right), 1 \leq k \leq K-1
$$


Particularly, when $k=K$, the transmission rate can be calculated by

$$
R_{b}^{K}=\log _{2}\left(1+\left|\mathbf{h}_{K} \mathbf{v}_{K}\right|^{2} /\left(I_{u}^{K}+\sigma^{2}\right)\right) .
$$

\section{B. Problem Formulation}

In this paper, our objective is to maximize the sum rate of all ground users in the cell by jointly optimizing user scheduling $\mathbf{S}=\left\{s_{i}[n], \forall i \in \mathcal{I}, \forall n\right\}$, UAV trajectory $\mathbf{W}=\{\mathbf{w}[n], \forall n\}$ and NOMA precoding vectors $\mathbf{V}=\left\{\mathbf{v}_{k}, \forall k \in \mathcal{K}\right\}$, with a minimum rate threshold $\eta$ predefined for users served by UAV, i.e., $R_{u}^{i} \geq$ $\eta, \forall i \in \mathcal{I}$. In addition, the interference from the UAV to users served by the BS are constrained to satisfy $I_{u}^{k} \leq \gamma, \forall k \in \mathcal{K}$, and the interference from BS to the UAV-served user is zeroforced in each time slot with sufficient antennas equipped at the BS, i.e., $I_{b}^{i}=0$ for $s_{i}[n]=1$. Thus, (12) can be changed into

$$
\gamma \leq\left|\mathbf{h}_{k} \mathbf{v}_{K}\right|^{2} \leq\left|\mathbf{h}_{k} \mathbf{v}_{K-1}\right|^{2} \leq \cdots \leq\left|\mathbf{h}_{k} \mathbf{v}_{1}\right|^{2}, \forall k \in \mathcal{K} .
$$

With the aforementioned objective and contraints, the joint optimization problem can be formulated as

$$
\begin{aligned}
\max _{\mathbf{S}, \mathbf{W}, \mathbf{V}} & R^{\text {sum }} \\
\text { s.t. } & R_{u}^{i} \geq \eta, \forall i \in \mathcal{I} \\
& I_{u}^{k} \leq \gamma, \forall k \in \mathcal{K}, \\
& \mathbf{w}[1]=\mathbf{w}[\mathcal{N}], \\
& \|\mathbf{w}[n+1]-\mathbf{w}[n]\|^{2} \leq(v \mathcal{T} / \mathcal{N})^{2}, n=1, \ldots, \mathcal{N}-1, \\
& s_{i}[n]=\{0,1\}, \forall i \in \mathcal{I}, \forall n \\
& \sum_{i \in \mathcal{I}} s_{i}[n] \leq 1, \forall n, \\
& \gamma \leq\left|\mathbf{h}_{k} \mathbf{v}_{K}\right|^{2} \leq\left|\mathbf{h}_{k} \mathbf{v}_{K-1}\right|^{2} \leq \ldots \leq\left|\mathbf{h}_{k} \mathbf{v}_{1}\right|^{2}, \forall k \in \mathcal{K}, \\
& I_{b}^{i}=0, s_{i}[n]=1, i \in \mathcal{I}, \\
& \sum_{k=1}^{K}\left\|\mathbf{v}_{k}\right\|^{2} \leq P_{t h}, k \in \mathcal{K},
\end{aligned}
$$

where $R^{\text {sum }}$ denotes the sum rate of all users in the network and $P_{t h}$ is the upper bound of the BS transmit power. From the joint optimization, we can observe that constraints (16b) and (16i) guarantee the performances of users served by UAV while constraint (16c) considers the QoS of BS-served users. In addition, the UAV trajectory restrictions (16d) and (16e), user scheduling constraints (16f) and (16g), NOMA decoding condition (16h), and the BS transmit power constraint (16j) are all involved. Specifically, the objective function $R^{\text {sum }}$ can be divided into two parts as

$$
R^{s u m}=R_{u}^{s u m}+R_{b}^{s u m}=\sum_{i \in \mathcal{I}} R_{u}^{i}+\sum_{k \in \mathcal{K}} R_{b}^{k},
$$

according to (9), (13) and (14). The joint problem in (16) has both combinatorial and continuous variables, which is nonconvex. Motivated by (17), this problem can be separated into two subproblems to solve. First, we will study the UAV trajectory optimization in Section III to maximize the sum rate of UAV-served users by jointly optimizing $\mathbf{S}$ and $\mathbf{W}$. Then, based on the optimized $\mathbf{S}$ and $\mathbf{W}$, we will concentrate on the joint precoding optimization of the NOMA network in Section
IV to maximize the sum rate of BS-served users.

\section{UAV TRAJECTORY OPTIMIZATION}

In this section, we aim to maximize the throughput of all the UAV-served users via jointly optimizing the trajectory and scheduling, with a limitation on the interference from the UAV to the BS-served users. First, the problem formulation is presented. Then, we apply the method of block coordinate descent to optimize the user scheduling $\mathbf{S}$ and UAV trajectory W alternatively. Finally, the proposed alternating optimization algorithm is summarized.

\section{A. Problem Formulation}

From (16), the optimization of UAV can be given by

$$
\begin{aligned}
\max _{\mathbf{S}, \mathbf{w}} & R_{u}^{\text {sum }} \\
\text { s.t. } & R_{u}^{i} \geq \eta, \forall i \in \mathcal{I} \\
& I_{u}^{k} \leq \gamma, \forall k \in \mathcal{K} \\
& \mathbf{w}[1]=\mathbf{w}[\mathcal{N}] \\
& \|\mathbf{w}[n+1]-\mathbf{w}[n]\|^{2} \leq(v \mathcal{T} / \mathcal{N})^{2}, n=1, \ldots, \mathcal{N}-1 \\
& s[n]=\{0,1\}, \forall i \in \mathcal{I}, \forall n \\
& \sum_{i \in \mathcal{I}} s_{i}[n] \leq 1, \forall n
\end{aligned}
$$

Assume that the interference from the BS to the UAV-served user can be zero-forced in each time slot via NOMA precoding, and thus constraint (18b) is equivalent as

$$
\begin{aligned}
R_{u}^{i} & =\frac{1}{\mathcal{N}} \sum_{n=1}^{\mathcal{N}} s_{i}[n] \log _{2}\left(1+\frac{\frac{p \rho_{u}}{H^{2}+\left\|\mathbf{w}[n]-\mathbf{L}_{i}\right\|^{2}}}{I_{b}^{i}+\sigma^{2}}\right) \\
& =\frac{1}{\mathcal{N}} \sum_{n=1}^{\mathcal{N}} s_{i}[n] \log _{2}\left(1+\frac{p \rho_{u}}{\sigma^{2}\left(H^{2}+\left\|\mathbf{w}[n]-\mathbf{L}_{i}\right\|^{2}\right)}\right) \geq \eta .
\end{aligned}
$$

Different from [29], the performance of BS-served users is also considered in (18c).

The problem in (18) is difficult to solve as it is a mixedinteger non-convex problem. Thus, we relax the binary variables $s_{i}[n]$ into continuous ones, i.e., $0 \leq \tilde{s}_{i}[n] \leq 1, \forall n, \forall i \in \mathcal{I}$. The relaxed problem is still non-convex and cannot be solved directly. To solve this problem effectively, it will be recast into two subproblems using block coordinate descent.

\section{B. Subproblem 1: Scheduling Optimization}

For any given UAV trajectory $\mathbf{W}$, the transmission scheduling problem can be expressed as ${ }^{2}$

$$
\begin{array}{ll}
\max _{\mathbf{S}} & R_{u}^{\text {sum }} \\
\text { s.t. } & R_{u}^{i} \geq \eta, \forall i \in \mathcal{I}, \\
& 0 \leq \tilde{s}_{i}[n] \leq 1, \forall n, \forall i \in \mathcal{I}, \\
& \sum_{i \in \mathcal{I}} \tilde{s}_{i}[n] \leq 1, \forall n .
\end{array}
$$

\footnotetext{
${ }^{2}$ Although the user scheduling can also be solved by exhaustive searching, its complexity is extremely high to find the optimal solution among $\mathcal{I}^{\mathcal{N}}$ possible candidates.
} 
The problem (20) is a standard linear programming problem, which can be solved by classical optimization tools such as CVX.

\section{Subproblem 2: Trajectory Optimization}

With the transmission scheduling $\mathbf{S}$ obtained by solving (20), the UAV trajectory optimization can be formulated as

$$
\begin{aligned}
\max _{\mathbf{w}} & \sum_{i \in \mathcal{I}} R_{u}^{i} \\
\text { s.t. } & \frac{1}{\mathcal{N}} \sum_{n=1}^{\mathcal{N}} \tilde{s}_{i}[n] \log _{2}\left(1+\frac{\frac{p \rho_{u}}{H^{2}+\left\|\mathbf{w}[n]-\mathbf{L}_{i}\right\|^{2}}}{\sigma^{2}}\right) \geq \eta, \forall i \in \mathcal{I}, \\
& \frac{p \rho_{u}}{H^{2}+\left\|\mathbf{w}[n]-\mathbf{L}_{k}\right\|^{2}} \leq \gamma, \forall k \in \mathcal{K}, \\
& \mathbf{w}[1]=\mathbf{w}[\mathcal{N}], \\
& \|\mathbf{w}[n+1]-\mathbf{w}[n]\|^{2} \leq(v \mathcal{T} / \mathcal{N})^{2}, n=1, \ldots, \mathcal{N}-1 .
\end{aligned}
$$

The problem (21) is intractable due to the non-convex objective function (21a) and constraints (21b) and (21c). Thus, the successive convex optimization technique can be adopted to approximate it as a convex one.

First, the rate of the $i$ th user served by the UAV in the $n$th time slot can be expressed as

$$
\widetilde{R}_{u}^{i}[n]=\log _{2}\left(1+\frac{p \rho_{u}}{\sigma^{2}\left(H^{2}+\left\|\mathbf{w}[n]-\mathbf{L}_{i}\right\|^{2}\right)}\right),
$$

which is not concave with respect to $\mathbf{w}[n]$, but is convex with respect to $\left\|\mathbf{w}[n]-\mathbf{L}_{i}\right\|^{2}$. Since a convex function is globally lower-bounded by its first-order Taylor expansion at any local point, we can obtain the lower bound $\widetilde{R}_{u}^{i}[n]$ with given UAV trajectory $\mathbf{W}^{r}$ in the $(r+1)$ th iteration as

$$
\begin{aligned}
\widetilde{R}_{u}^{i}[n] & \geq-X_{i}^{r}[n]\left(\left\|\mathbf{w}[n]-\mathbf{L}_{i}\right\|^{2}-\left\|\mathbf{w}^{r}[n]-\mathbf{L}_{i}\right\|^{2}\right)+Y_{i}^{r}[n] \\
& \triangleq R_{u l}^{i}[n],
\end{aligned}
$$

where both $X_{i}^{r}[n]$ and $Y_{i}^{r}[n]$ are constants calculated as

$$
\begin{gathered}
X_{i}^{r}[n]=\frac{\frac{p \rho_{u}}{\sigma^{2}\left(H^{2}+\left\|\mathbf{w}^{r}[n]-\mathbf{L}_{i}\right\|^{2}\right)^{2}} \log _{2}(e)}{1+\frac{p \rho_{u}}{\sigma^{2}\left(H^{2}+\left\|\mathbf{w}^{r}[n]-\mathbf{L}_{i}\right\|^{2}\right)}}, \\
Y_{i}^{r}[n]=\log _{2}\left(1+\frac{p \rho_{u}}{\sigma^{2}\left(H^{2}+\left\|\mathbf{w}^{r}[n]-\mathbf{L}_{i}\right\|^{2}\right)}\right) .
\end{gathered}
$$

Thus, the lower bound $R_{u l}^{i}[n]$ is concave with respect to $\mathbf{w}[n]$ and (21b) can be approximated as

$$
\frac{1}{\mathcal{N}} \sum_{n=1}^{\mathcal{N}} \tilde{s}_{i}[n] R_{u l}^{i}[n] \geq \eta, \forall i \in \mathcal{I} .
$$

For the non-convex constraint (21c), similarly, we can adopt the first-order Taylor expansion at the given local point $\mathbf{W}^{r}$ to give

$$
\left\|\mathbf{w}[n]-\mathbf{L}_{k}\right\|^{2} \geq\left\|\mathbf{w}^{r}[n]-\mathbf{L}_{k}\right\|^{2}+2\left(\mathbf{w}^{r}[n]-\mathbf{L}_{k}\right)^{T}\left(\mathbf{w}[n]-\mathbf{w}^{r}[n]\right) .
$$

Thus, the constraint (21c) can be approximated as

$$
H^{2}+\left\|\mathbf{w}^{r}[n]-\mathbf{L}_{k}\right\|^{2}+2\left(\mathbf{w}^{r}[n]-\mathbf{L}_{k}\right)^{T}\left(\mathbf{w}[n]-\mathbf{w}^{r}[n]\right) \geq \frac{p \rho_{u}}{\gamma},
$$

which is concave with respect to $\mathbf{w}[n]$. Using (26) and (28) and the given transmission scheduling $\mathbf{S}$, the original problem
(21) can be approximated as

$$
\begin{aligned}
\max _{\mathbf{W}} & \sum_{i \in \mathcal{I}}\left(\frac{1}{\mathcal{N}} \sum_{n=1}^{\mathcal{N}} \tilde{s}_{i}[n] R_{u l}^{i}[n]\right) \\
\text { s.t. } & \frac{1}{\mathcal{N}} \sum_{n=1}^{\mathcal{N}} \tilde{s}_{i}[n] R_{u l}^{i}[n] \geq \eta, \forall i \in \mathcal{I}, \\
& H^{2}+\left\|\mathbf{w}^{r}[n]-\mathbf{L}_{k}\right\|^{2}+2\left(\mathbf{w}^{r}[n]-\mathbf{L}_{k}\right)^{T}\left(\mathbf{w}[n]-\mathbf{w}^{r}[n]\right) \\
& \geq \frac{p \rho_{u}}{\gamma}, \forall k \in \mathcal{K}, \\
& \mathbf{w}[1]=\mathbf{w}[\mathcal{N}], \\
& \|\mathbf{w}[n+1]-\mathbf{w}[n]\|^{2} \leq(v \mathcal{T} / \mathcal{N})^{2}, n=1, \ldots, \mathcal{N}-1 .
\end{aligned}
$$

It can be observed that the objective function (29a) as well as constraint (29b) are convex now, since $R_{u l}^{i}[n]$ is concave with respect to $\mathbf{w}[n]$. In addition, the left side expression in constraint (29c) is also concave with respect to $\mathbf{w}[n]$, so the constraint is convex. Therefore, the problem (29) is a convex optimization problem which can be solved by CVX effectively.

\section{Alternating Optimization Algorithm}

The above alternating optimization can be summarized as Algorithm 1.

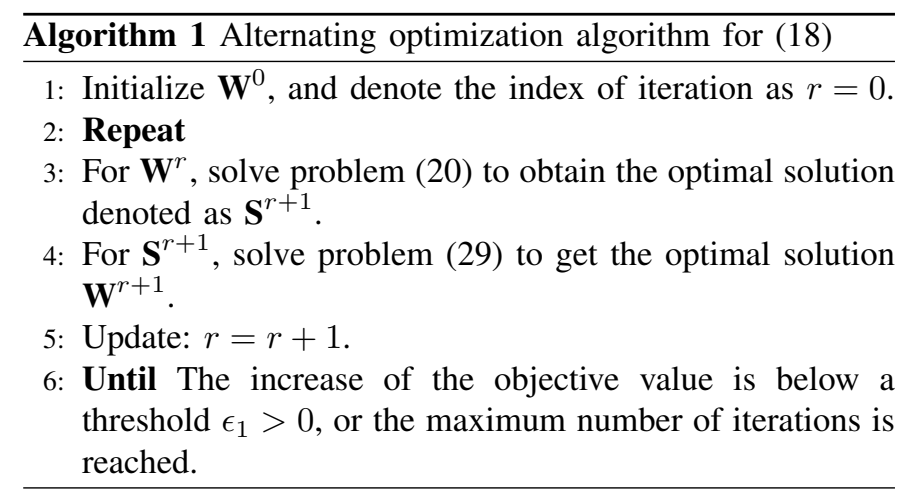

In the algorithm, user scheduling $\mathbf{S}$ and UAV trajectory W are optimized alternately by solving (20) and (29). The convergence of the algorithm is demonstrated as follows.

Remark 1: With given $\left\{\mathbf{S}^{r}, \mathbf{W}^{r}\right\}$, the solution $\left\{\mathbf{S}^{r+1}, \mathbf{W}^{r}\right\}$ obtained in the $(r+1)$ th iteration by solving problem (20) is optimal and the objective value is non-decreasing with iterations. On the other hand, for given $\left\{\mathbf{S}^{r+1}, \mathbf{W}^{r}\right\}$, the obtained solution $\left\{\mathbf{S}^{r+1}, \mathbf{W}^{r+1}\right\}$ in the $(r+1)$ th iteration by solving the approximate problem (29) is optimal and the objective value is a lower bound of that for its original problem (21). Thus, the objective value is non-decreasing after each iteration of Algorithm 1. Furthermore, the objective value of the problem (18) is upper bounded by a finite value, and thus Algorithm 1 is guaranteed to converge with a polynomial complexity. Due to the approximate conversions, the obtained solution by Algorithm 1 is a sub-optimal one of the original problem (18). Notably, the performance of the algorithm depends on the initial UAV trajectory. We adopt a simple circular trajectory with the geometric center of users as the circle center, i.e., $\mathbf{C}_{u}=\sum_{i=1}^{I} \mathbf{L}_{i} / I$, and the radius obtained as $\min \left(\frac{v \mathcal{T}}{2 \pi},\left\|\mathbf{L}_{i}-\mathbf{C}_{u}\right\|\right)$. 
After the convergence of Algorithm 1, the user scheduling variables $\tilde{s}_{i}[n]$ obtained are usually tight and nearly binary ones. Otherwise, each time slot will be further divided into $\delta$ sub-slots, $\delta \geq 1$, and the number of sub-slots assigned to the $i$ th user in the $n$th time slot is denoted as $N_{i}[n]=\delta \tilde{s}_{i}[n]$. Then, we can set $\delta$ large enough to make the values of $N_{i}[n]$ all integers according to [29].

In addition, according to the user scheduling in the scheme, the UAV can serve each user for several continuous time slots. In this case, the radio control connection time can be ignored, because it is only needed in the beginning slot when the UAV serves a specific user.

\section{JOINT PRECODING FOR NOMA}

In this section, two schemes are proposed to jointly optimize the precoding vectors at the NOMA BS [38], [39]. In the first scheme, the precoding vectors are optimized to maximize the sum rate of the BS-served users with the interference to the UAV-served user in each time slot zero-forced. While in the second one, the precoding vectors are optimized to maximize the sum rate of both the BS-served users and the UAV-served user in each time slot, with the power of interference to the UAV-served users constrained.

\section{A. Scheme I: Zero-forcing at UAV}

In the first scheme, our goal is to maximize the sum rate of BS-served users by jointly optimizing the precoding vectors, with the decoding order satisfied and the interference from the BS to the UAV-served user zero-forced. For any given transmission scheduling and UAV trajectory $\{\mathbf{S}, \mathbf{W}\}$, the precoding optimization problem can be formulated as

$$
\begin{aligned}
\max _{\mathbf{v}_{k}} & R_{b}^{\text {sum }} \\
\text { s.t. } & \gamma \leq\left|\mathbf{h}_{k} \mathbf{v}_{K}\right|^{2} \leq\left|\mathbf{h}_{k} \mathbf{v}_{K-1}\right|^{2} \leq \ldots \leq\left|\mathbf{h}_{k} \mathbf{v}_{1}\right|^{2}, \forall k \in \mathcal{K}, \\
& \mathbf{h}_{i} \mathbf{v}_{k}=0, \forall k \in \mathcal{K}, s_{i}[n]=1, i \in \mathcal{I}, \\
& \sum_{k=1}^{K}\left\|\mathbf{v}_{k}\right\|^{2} \leq P_{t h}, k \in \mathcal{K},
\end{aligned}
$$

where $\gamma$ is the maximum power of the interference generated by UAV. The constraint (30c) indicates that the interference from the BS should be zero-forced at the specific UAVserved user $i$ in the $n$th time slot according to the optimized transmission scheduling of UAV, which can guarantee the performance of $R^{\text {sum }}$ in (16) accordingly. We can observe that the problem (30) is non-convex, which cannot be solved directly. Particularly, the objective function (30a) and the constraint (30b) are both non-convex, and thus it is necessary to transform them first.

To perform the approximate transformations, we introduce some auxiliary variables $r_{k}, k \in \mathcal{K}$, to reformulate (30) according to (13) and (14) as

$$
\begin{array}{ll}
\max _{\mathbf{v}_{k}, r_{k}} & \log _{2}\left(r_{1} r_{2} \ldots r_{K}\right) \\
\text { s.t. } & 1+\frac{\left|\mathbf{h}_{k} \mathbf{v}_{k}\right|^{2}}{\sum_{j=k+1}^{K}\left|\mathbf{h}_{k} \mathbf{v}_{j}\right|^{2}+I_{u}^{k}+\sigma^{2}} \geq r_{k}, k=1, \ldots, K-1 \\
& 1+\frac{\left|\mathbf{h}_{K} \mathbf{v}_{K}\right|^{2}}{I_{u}^{K}+\sigma^{2}} \geq r_{K} \\
& \gamma \leq\left|\mathbf{h}_{k} \mathbf{v}_{K}\right|^{2} \leq\left|\mathbf{h}_{k} \mathbf{v}_{K-1}\right|^{2} \leq \ldots \leq\left|\mathbf{h}_{k} \mathbf{v}_{1}\right|^{2}, \forall k \in \mathcal{K} \\
& \mathbf{h}_{i} \mathbf{v}_{k}=0, \forall k \in \mathcal{K}, s_{i}[n]=1, i \in \mathcal{I} \\
& \sum_{k=1}^{K}\left\|\mathbf{v}_{k}\right\|^{2} \leq P_{t h}, k \in \mathcal{K}
\end{array}
$$

One sees that the logarithmic function in (31a) is nondecreasing, for which the objective function can be equivalent to maximize the geometric mean among $r_{k}$, i.e., $\left(\prod_{k=1}^{K} r_{k}\right)^{1 / K}$, which is concave and increasing. Since the geometric mean can be recast as a series of second-order-cone (SOC) constraints and then solved efficiently by classical optimization methods, the problem (31) can be changed into

$$
\begin{aligned}
\max _{\mathbf{v}_{k}, r_{k}} & \left(\prod_{k=1}^{K} r_{k}\right)^{\frac{1}{K}} \\
\text { s.t. } & \sum_{j=k+1}^{K}\left|\mathbf{h}_{k} \mathbf{v}_{j}\right|^{2}+I_{u}^{k}+\sigma^{2} \leq \frac{\left|\mathbf{h}_{k} \mathbf{v}_{k}\right|^{2}}{r_{k}-1}, k=1, \ldots, K-1,(12 t) \\
& I_{u}^{K}+\sigma^{2} \leq\left|\mathbf{h}_{K} \mathbf{v}_{K}\right|^{2} /\left(r_{K}-1\right) \\
& \gamma \leq\left|\mathbf{h}_{k} \mathbf{v}_{K}\right|^{2} \leq\left|\mathbf{h}_{k} \mathbf{v}_{K-1}\right|^{2} \leq \ldots \leq\left|\mathbf{h}_{k} \mathbf{v}_{1}\right|^{2}, \forall k \in \mathcal{K} \\
& \mathbf{h}_{i} \mathbf{v}_{k}=0, \forall k \in \mathcal{K}, s_{i}[n]=1, i \in \mathcal{I} \\
& \sum_{k=1}^{K}\left\|\mathbf{v}_{k}\right\|^{2} \leq P_{t h}, k \in \mathcal{K}
\end{aligned}
$$

It can be observed that the problem (32) is still intractable as (32b), (32c) and (32d) are not convex. Some further approximations are still needed.

First, we define the following functions.

$$
\begin{gathered}
F_{k}\left(\mathbf{v}_{k}, r_{k}\right)=\left|\mathbf{h}_{k} \mathbf{v}_{k}\right|^{2} /\left(r_{k}-1\right), \\
F_{k j}\left(\mathbf{v}_{j}\right)=\left|\mathbf{h}_{k} \mathbf{v}_{j}\right|^{2} .
\end{gathered}
$$

(33) is convex in a quadratic-over-line form and (34) is convex with respect to $\mathbf{v}_{j}$. Thus, the two real-valued functions can be approximated by their corresponding first-order Taylor expansions over a certain point [40], which hold

$$
\begin{aligned}
& F_{k}\left(\mathbf{v}_{k}, r_{k}\right) \geq F_{k}\left(\mathbf{v}_{k}^{i}, r_{k}^{i}\right)+2 \operatorname{Re}\left\{\frac{\partial F_{k}\left(\mathbf{v}_{k}, r_{k}\right)}{\partial \mathbf{v}_{k}^{i}}\left(\mathbf{v}_{k}-\mathbf{v}_{k}^{i}\right)\right\} \\
&+\frac{\partial F_{k}\left(\mathbf{v}_{k}, r_{k}\right)}{\partial r_{k}^{i}}\left(r_{k}-r_{k}^{i}\right)=\mathscr{T}_{k}\left(\mathbf{v}_{k}, r_{k}, \mathbf{v}_{k}^{i}, r_{k}^{i}\right), \\
& F_{k j}\left(\mathbf{v}_{j}\right) \geq F_{k j}\left(\mathbf{v}_{j}^{i}\right)+2 \operatorname{Re}\left\{\frac{\partial F_{k j}\left(\mathbf{v}_{j}\right)}{\partial \mathbf{v}_{j}^{i}}\left(\mathbf{v}_{j}-\mathbf{v}_{j}^{i}\right)\right\} \\
&=\mathscr{T}_{k j}\left(\mathbf{v}_{j}, \mathbf{v}_{j}^{i}\right) .
\end{aligned}
$$

Due to the fact that $\mathbf{h}_{k} \mathbf{v}_{k}^{i} \mathbf{v}_{k}^{i \dagger} \mathbf{h}_{k}^{\dagger}=\operatorname{Re}\left(\mathbf{h}_{k} \mathbf{v}_{k}^{i} \mathbf{v}_{k}^{i \dagger} \mathbf{h}_{k}^{\dagger}\right)$ and 


$$
\begin{aligned}
\max _{\mathbf{v}_{k}, r_{k}} & t^{0} \\
\text { s.t. } & \left\|\left[2 t_{j}^{\mathcal{C}-1},\left(r_{2 j-1}-r_{2 j}\right)\right]^{\dagger}\right\| \leq r_{2 j-1}+r_{2 j}, j=1,2, \ldots, 2^{\mathcal{C}-1}, \\
& \left\|\left[2 t_{j}^{\mathcal{C}-2},\left(t_{2 j-1}^{\mathcal{C}-1}-t_{2 j}^{\mathcal{C}-1}\right)\right]^{\dagger}\right\| \leq t_{2 j-1}^{\mathcal{C}-1}+t_{2 j}^{\mathcal{C}-1}, j=1,2, \ldots, 2^{\mathcal{C}-2}, \\
& \ldots . \\
& \left\|\left[2 t_{1}^{0},\left(t_{1}^{1}-t_{2}^{1}\right)\right]^{\dagger}\right\| \leq t_{1}^{1}+t_{2}^{1}, j=1, \\
& \left\|\left[2 \mathbf{h}_{k} \mathbf{v}_{k+1}, \ldots, 2 \mathbf{h}_{k} \mathbf{v}_{K-1}, 2 \mathbf{h}_{k} \mathbf{v}_{K}, 2 \sqrt{I_{u}^{k}}, 2 \sigma,\left(\mathscr{T}_{k}-1\right)\right]^{\dagger}\right\| \leq \mathscr{T}_{k}+1, k=1,2, \ldots, K-1, \\
\| & {\left[\left[2 \sqrt{I_{u}^{K}}, 2 \sigma,\left(\mathscr{T}_{K}-1\right)\right]^{\dagger} \| \leq \mathscr{T}_{K}+1,\right.} \\
\| & {[n] \mathbf{h}_{i} \mathbf{v}_{k}=0, \forall k \in \mathcal{K}, \forall i \in \mathcal{I}, } \\
& \mathcal{H} \text { and }\left\|\left[\mathbf{v}_{1}^{\dagger}, \mathbf{v}_{2}^{\dagger}, \ldots, \mathbf{v}_{K}^{\dagger}\right]^{\dagger}\right\| \leq \sqrt{P_{t h}} .
\end{aligned}
$$

$\mathbf{h}_{k} \mathbf{v}_{j}^{i} \mathbf{v}_{j}^{i \dagger} \mathbf{h}_{k}^{\dagger}=R e\left(\mathbf{h}_{k} \mathbf{v}_{j}^{i} \mathbf{v}_{j}^{i \dagger} \mathbf{h}_{k}^{\dagger}\right)$, the first-order Taylor approximations $\mathscr{T}_{k}\left(\mathbf{v}_{k}, r_{k}, \mathbf{v}_{k}^{i}, r_{k}^{i}\right)$ and $\mathscr{T}_{k j}\left(\mathbf{v}_{j}, \mathbf{v}_{j}^{i}\right)$ are calculated as

$$
\begin{gathered}
\mathscr{T}_{k}\left(\mathbf{v}_{k}, r_{k}, \mathbf{v}_{k}^{i}, r_{k}^{i}\right)=2 \operatorname{Re}\left(\mathbf{h}_{k} \mathbf{v}_{k}^{i} \mathbf{v}_{k}^{\dagger} \mathbf{h}_{k}^{\dagger}\right) /\left(r_{k}^{i}-1\right) \\
-\mathbf{h}_{k} \mathbf{v}_{k}^{i} \mathbf{v}_{k}^{i \dagger} \mathbf{h}_{k}^{\dagger}\left(r_{k}-1\right) /\left(r_{k}^{i}-1\right)^{2}, \\
\mathscr{T}_{k j}\left(\mathbf{v}_{j}, \mathbf{v}_{j}^{i}\right)=2 \operatorname{Re}\left(\mathbf{h}_{k} \mathbf{v}_{j} \mathbf{v}_{j}^{i \dagger} \mathbf{h}_{k}^{\dagger}\right)-\mathbf{h}_{k} \mathbf{v}_{j}^{i} \mathbf{v}_{j}^{i \dagger} \mathbf{h}_{k}^{\dagger},
\end{gathered}
$$

where $\mathbf{h}_{k}^{\dagger} \mathbf{h}_{k} \succeq 0$ and $r_{k}>1$. Then, the non-convex constraints (32b) and (32c) can be converted into convex ones via substituting the right-side functions with their approximations derived above.

As for the non-convex constraint (32d), the following expressions are utilized to indicate the decoding order of the $k$ th user, $k \in \mathcal{K}$.

$$
\mathcal{H}_{k}=\left\{\begin{array}{l}
\gamma \leq\left|\mathbf{h}_{k} \mathbf{v}_{K}\right|^{2} \\
\left|\mathbf{h}_{k} \mathbf{v}_{K}\right|^{2} \leq \min \left\{\left|\mathbf{h}_{k} \mathbf{v}_{K-1}\right|^{2}, \ldots,\left|\mathbf{h}_{k} \mathbf{v}_{1}\right|^{2}\right\} \\
\left|\mathbf{h}_{k} \mathbf{v}_{K-1}\right|^{2} \leq \min \left\{\left|\mathbf{h}_{k} \mathbf{v}_{K-2}\right|^{2}, \ldots,\left|\mathbf{h}_{k} \mathbf{v}_{1}\right|^{2}\right\} \\
\ldots \ldots, \\
\left|\mathbf{h}_{k} \mathbf{v}_{2}\right|^{2} \leq\left|\mathbf{h}_{k} \mathbf{v}_{1}\right|^{2}
\end{array}\right.
$$

Then, by substituting the item $\left|\mathbf{h}_{k} \mathbf{v}_{k}\right|^{2}$ with its approximation expressed in (38), the constraint (39) can be rewritten as

$$
\widetilde{\mathcal{H}}_{k}=\left\{\begin{array}{l}
\gamma \leq \mathscr{T}_{k K}\left(\mathbf{v}_{K}, \mathbf{v}_{K}^{i}\right) \\
\left|\mathbf{h}_{k} \mathbf{v}_{K}\right|^{2} \leq \min _{j \in[1, K-1]}\left\{\mathscr{T}_{k j}\left(\mathbf{v}_{j}, \mathbf{v}_{j}^{i}\right)\right\} \\
\left|\mathbf{h}_{k} \mathbf{v}_{K-1}\right|^{2} \leq \min _{j \in[1, K-2]}\left\{\mathscr{T}_{k j}\left(\mathbf{v}_{j}, \mathbf{v}_{j}^{i}\right)\right\} \\
\ldots \ldots \ldots, \\
\left|\mathbf{h}_{k} \mathbf{v}_{2}\right|^{2} \leq \mathscr{T}_{k 1}\left(\mathbf{v}_{1}, \mathbf{v}_{1}^{i}\right) .
\end{array}\right.
$$

For simplicity, we utilize $\mathcal{H} \triangleq\left(\widetilde{\mathcal{H}}_{1}, \widetilde{\mathcal{H}}_{2}, \ldots, \widetilde{\mathcal{H}}_{K}\right)$ to denote the transformed form of the decoding constraint (32d), which is convex now.

For the objective function, the geometric mean can be reformulated as a second-order cone programming (SOCP) because the hyperbolic constraint $z^{2} \leq x y(x \geq 0, y \geq 0)$ will result in $\left\|[2 z, x-y]^{\dagger}\right\| \leq x+y$. Similarly, the constraints (32b) and (32c) can also be transformed to a series of SOC constraints based on this relationship. With all these derivations completed, the original problem (30) can be recast to a SOCP problem (41) at the top of this page, which is convex and much easier to solve. In (41), $\mathcal{C}=\left\lceil\log _{2} K\right\rceil$, which is a ceiling function and returns the smallest integer no less than $\log _{2} K$. In addition, we define $r_{i}=1$ for the case $K<2^{\mathcal{C}}$, where $i=K+1, \ldots, 2^{\left\lceil\log _{2} K\right\rceil}$.

With the optimized $\{\mathbf{S}, \mathbf{W}\}$ obtained by UAV trajectory optimization in Section III and given points $\left(\mathbf{v}_{k}^{i}, r_{k}^{i}\right)$ in the $i$ th iteration, the SOCP problem (41) can be solved effectively by utilizing existing convex optimization tools such as CVX. Considering the series of approximate transformations derived above, an iterative algorithm is proposed to obtain the suboptimal solution for the problem (30) via calculating this approximate optimization problem. Details of the proposed iterative algorithm are presented in Algorithm 2.

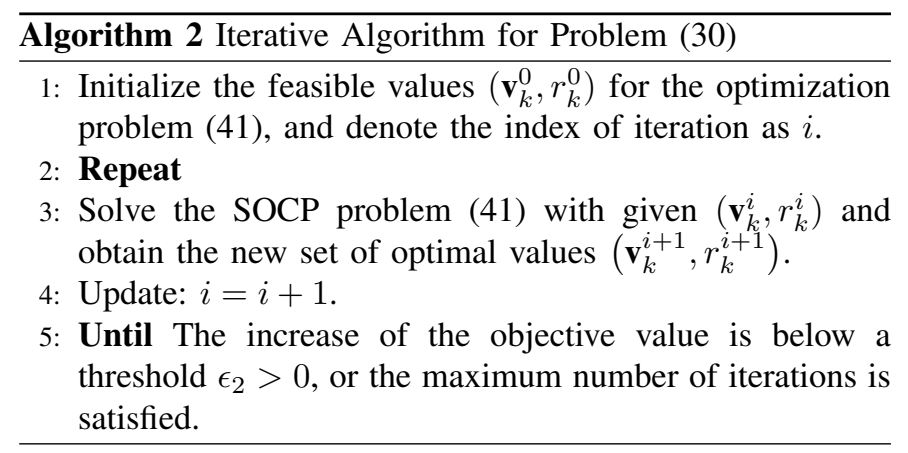

The initial values $\left(\mathbf{v}_{k}^{0}, r_{k}^{0}\right)$ can be generated randomly with the constraints in (41) considered, which can be obtained easily in practice [12].

Remark 2: It's worth pointing out that the objective value acquired after each iteration is no less than that of the prior iteration. This indicates that the sum rate is non-decreasing with iterations. The transmit power constraint and decoding requirements make a upper bound for the sum rate, which 


$$
\begin{array}{ll}
\max _{\mathbf{v}_{k}, r_{k}} t^{0} & \left\|\left[2 t_{j}^{\widetilde{\mathcal{C}}-1},\left(r_{2 j-1}-r_{2 j}\right)\right]^{\dagger}\right\| \leq r_{2 j-1}+r_{2 j}, j=1,2, \ldots, 2^{\widetilde{\mathcal{C}}-1}, \\
\text { s.t. } & \left\|\left[2 t_{j}^{\widetilde{\mathcal{C}}-2},\left(t_{2 j-1}^{\widetilde{\mathcal{C}}-1}-t_{2 j}^{\widetilde{\mathcal{C}}-1}\right)\right]^{\dagger}\right\| \leq t_{2 j-1}^{\widetilde{\mathcal{C}}-1}+t_{2 j}^{\widetilde{\mathcal{C}}-1}, j=1,2, \ldots, 2^{\widetilde{\mathcal{C}}-2}, \\
& \ldots\left[2 t_{1}^{0},\left(t_{1}^{1}-t_{2}^{1}\right)\right]^{\dagger} \| \leq t_{1}^{1}+t_{2}^{1}, j=1, \\
& \left\|\left[2 \mathbf{h}_{k} \mathbf{v}_{k+1}, \ldots, 2 \mathbf{h}_{k} \mathbf{v}_{K-1}, 2 \mathbf{h}_{k} \mathbf{v}_{K}, 2 \sqrt{I_{u}^{k}}, 2 \sigma,\left(\mathscr{T}_{k}-1\right)\right]^{\dagger}\right\| \leq \mathscr{T}_{k}+1, k=1,2, \ldots, K-1, \\
& \left\|\left[2 \sqrt{I_{u}^{K}}, 2 \sigma,\left(\mathscr{T}_{K}-1\right)\right]^{\dagger}\right\| \leq \mathscr{T}_{K}+1, \\
& \left\|\left[2 \mathbf{h}_{i} \mathbf{v}_{1}, 2 \mathbf{h}_{i} \mathbf{v}_{2}, \ldots, 2 \mathbf{h}_{i} \mathbf{v}_{K}, 2 \sigma,\left(\mathscr{T}_{K+1}-1\right)\right]^{\dagger}\right\| \leq \mathscr{T}_{K+1}+1, s_{i}[n]=1, i \in \mathcal{I}, \\
& \left\|\left[\mathbf{h}_{i} \mathbf{v}_{1}, \mathbf{h}_{i} \mathbf{v}_{2}, \ldots, \mathbf{h}_{i} \mathbf{v}_{K}\right]^{\dagger}\right\| \leq \sqrt{\beta}, s_{i}[n]=1, i \in \mathcal{I}, \\
& \mathcal{H} \text { and }\left\|\left[\mathbf{v}_{1}^{\dagger}, \mathbf{v}_{2}^{\dagger}, \ldots, \mathbf{v}_{K}^{\dagger}\right]^{\dagger}\right\| \leq \sqrt{P_{t h}} .
\end{array}
$$

will guarantee the convergence of Algorithm 2. Although an approximate problem (41) is solved to maximize the sum rate, we can at least obtain a local optimum value for problem (30) or even a global optimal solution when proper initial values can be set.

\section{B. Scheme II: Interference Constrained at UAV}

In the first scheme, the interference from the BS should be perfectly zero-forced at each UAV-served user, which will result in the performance degradation of the BS-served users when the antennas at BS are not sufficient. Thus, in this subsection, the precoding vectors are jointly optimized to maximize the sum rate of both the BS-served users and the UAVserved user in each time slot, with the power of interference to the UAV-served user constrained. The optimization problem can be expressed as

$$
\begin{array}{ll}
\max _{\mathbf{v}_{k}} & R_{b}^{\text {sum }}+\widehat{R}_{u}^{i}[n] \\
\text { s.t. } & \gamma \leq\left|\mathbf{h}_{k} \mathbf{v}_{K}\right|^{2} \leq\left|\mathbf{h}_{k} \mathbf{v}_{K-1}\right|^{2} \leq \ldots \leq\left|\mathbf{h}_{k} \mathbf{v}_{1}\right|^{2}, \forall k \in \mathcal{K} \\
& \sum_{k=1}^{K}\left|\mathbf{h}_{i} \mathbf{v}_{k}\right|^{2} \leq \beta, s_{i}[n]=1, i \in \mathcal{I} \\
& \sum_{k=1}^{K}\left\|\mathbf{v}_{k}\right\|^{2} \leq P_{t h}, k \in \mathcal{K}
\end{array}
$$

where

$$
\widehat{R}_{u}^{i}[n]=\log _{2}\left(1+\frac{\frac{p \rho_{u}}{H^{2}+\left\|\mathbf{w}[n]-\mathbf{L}_{i}\right\|^{2}}}{\sum_{k \in \mathcal{K}}\left|\mathbf{h}_{i} \mathbf{v}_{k}\right|^{2}+\sigma^{2}}\right), s_{i}[n]=1, i \in \mathcal{I} .
$$

We can observe that the (30) and (42) have many similarities. The difference lies in the objective functions and the restriction on $\mathbf{h}_{i} \mathbf{v}_{k}$ in (30c) and (42c). Thus, the problem (42) can be transformed using similar approximate methods adopted in Section IV-A. A new auxiliary variable $r_{K+1}$ is defined as

$$
1+\frac{\frac{p \rho_{u}}{H^{2}+\left\|\mathbf{w}[n]-\mathbf{L}_{i}\right\|^{2}}}{\sum_{k \in \mathcal{K}}\left|\mathbf{h}_{i} \mathbf{v}_{k}\right|^{2}+\sigma^{2}} \geq r_{K+1}, s_{i}[n]=1 .
$$

In addition, we can exploit the first-order Taylor expansion at $r_{K+1}^{i}$ to derive

$$
\begin{aligned}
& \frac{\frac{p \rho_{u}}{H^{2}+\left\|\mathbf{w}[n]-\mathbf{L}_{i}\right\|^{2}}}{r_{K+1}-1} \\
\geq & \frac{p \rho_{u}}{H^{2}+\left\|\mathbf{w}[n]-\mathbf{L}_{i}\right\|^{2}} \\
r_{K+1}^{i}-1 & -\frac{p \rho_{u}}{H^{2}+\left\|\mathbf{w}[n]-\mathbf{L}_{i}\right\|^{2}} \\
\triangleq & \mathscr{T}_{K+1}\left(r_{K+1}^{i}, r_{K+1}^{i}\right) .
\end{aligned}
$$

Using all these approximations, (42) can be reformulated as (46), which is similar to (41). Particularly, the constant $\widetilde{\mathcal{C}}=$ $\left\lceil\log _{2}(K+1)\right\rceil$ and we define $r_{i}=1$ for the case $K+1<2^{\widetilde{\mathcal{C}}}$, where $i=K+2, \ldots, 2^{\left\lceil\log _{2}(K+1)\right\rceil}$. As a consequence, we can also solve the problem (46) efficiently using Algorithm 2 to get the sub-optimal solution for (42).

The key features of Scheme I and Scheme II are summarized as follows. In Scheme I, the interference from the BS to the $\mathrm{UAV}$-served user is zero-forced in each time slot, through which the performance of UAV-served users can be guaranteed free of interference. Nevertheless, when the antennas equipped at the BS are not sufficient, the performance of BS-served user$s$ may not be guaranteed. Thus, in Scheme II, the interference from the $\mathrm{BS}$ to the UAV-served user is constrained in each time slot instead of zero-forcing, and the performance of the BS-served users and the UAV-served users can be adjusted by properly changing the value of $\beta$. In addition, the convergence of the iterative algorithms for both Scheme I and Scheme II can be guaranteed, as demonstrated in Remark 2. Furthermore, both schemes are easily solved through the iterative algorithm, due to the convexity of (41) and (46). 


$$
\begin{aligned}
& \mathcal{O}\left(\sqrt{K^{2}+2 K+1+C_{1}}\left(2 K M+2 K+C_{1}-1\right)^{2}\left(0.5 K^{3}+K^{2}+4.5 K+K M+3 C_{1}-3\right)\right), \\
& \mathcal{O}\left(\sqrt{K^{2}+2 K+3+C_{2}}\left(2 K M+2 K+C_{2}+1\right)^{2}\left(0.5 K^{3}+K^{2}+5.5 K+K M+3 C_{2}+1\right)\right) .
\end{aligned}
$$

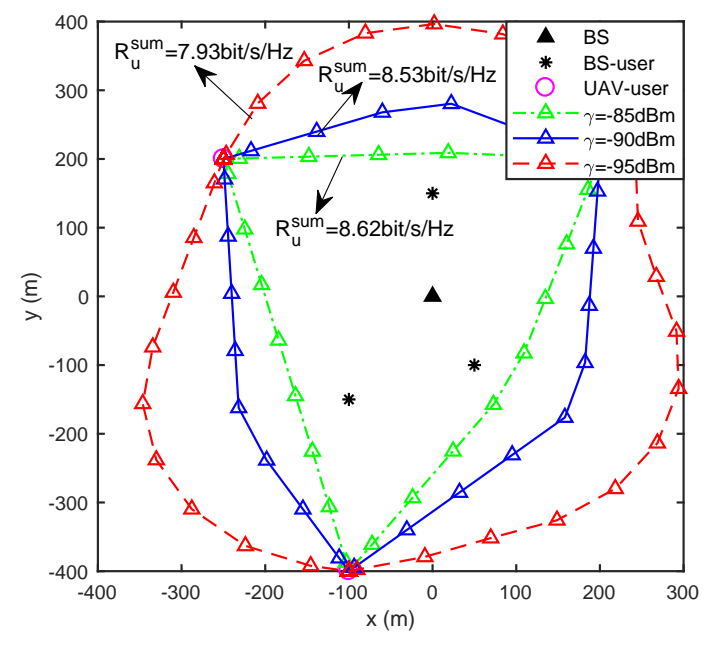

Fig. 2. Optimal UAV trajectories with different values of $\gamma . K=3$ and $I=3$. The UAV location of each time slot is marked by ' $\triangle$ '.

Remark 3: In both Scheme I and Scheme II, the solutions can be obtained by solving an SOCP problem in each iteration of Algorithm 2. Hence, according to [41], the computational complexity for (41) and (46) can be calculated as (47a) and (47b), respectively, where $C_{1}$ and $C_{2}$ are the non-negative integer constants caused by the equivalent SOC representations of the geometric means in the objective functions. From (47), we can observe that the complexity of Scheme II is a little higher than that of Scheme I, due to its larger number of variables and constraints.

\section{Simulation Results AND Discussion}

We consider a hybrid cellular network with a static BS and a flying UAV jointly serving several randomly distributed ground users, where $K$ users are served by the BS and the other $I$ users are served by the UAV. The UAV flies periodically at a fixed altitude $H=50 \mathrm{~m}$ above the area.

Firstly, $K=3 \mathrm{BS}$-served users and $I=3 \mathrm{UAV}$-served users are considered, following the topology in Fig. 2. The optimal UAV trajectories via Algorithm 1 are shown with different values of $\gamma . P_{t h}=20 \mathrm{dBm}$. According to the parameters for UAV communications in [28], [29], we set $\mathcal{N}=60, v=50$ $\mathrm{m} / \mathrm{s}, \mathcal{T}=100 \mathrm{~s}$ and $p=20 \mathrm{~mW}$. In addition, Scheme I of NOMA precoding is considered, in which the interference from the BS to the UAV-served user is zero-forced in each time slot. From Fig. 2, we can see that a triangle trajectory connecting three UAV-served users can be achieved when the allowable interference from the UAV to the BS-served users is $\gamma=-85 \mathrm{dBm}$, with highest $R_{u}^{\text {sum }}=8.62 \mathrm{bit} / \mathrm{s} / \mathrm{Hz}$. When $\gamma$ is smaller, the interference constraint (18c) to the BS-served users becomes stricter, and the UAV tends to fly away from the BS-served users to avoid interference. Accordingly, $R_{u}^{\text {sum }}$

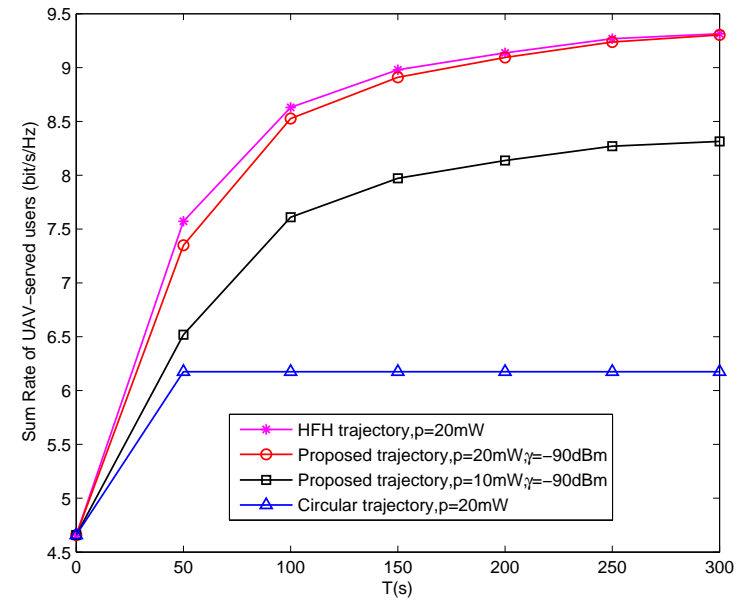

Fig. 3. Sum rate of UAV-served users with different trajectory schemes. $K=3$ and $I=3$.

of the UAV-served users becomes lower, due to the longer distance between UAV and its corresponding users.

In Fig. 3, we compare the sum rate of UAV-served users in the following schemes. 1) The proposed scheme as in Algorith$\mathrm{m} 1$;2) the hover-fly-hover (HFH) trajectory according to [36]; 3) the circular trajectory, whose initial trajectory is defined in Remark 1. For all these schemes, the user scheduling is jointly optimized with the corresponding trajectory. From the result, we can observe that the sum rate of circular trajectory does not increase when $\mathcal{T}$ is larger than $50 \mathrm{~s}$, while for the other two schemes, the sum rate increases with $\mathcal{T}$ and gets saturated when $\mathcal{T}$ is sufficiently large. Notably, the proposed scheme and the HFH trajectory significantly outperform the circular trajectory, and even with half of the transmit power, the proposed scheme still achieves a much higher rate than that of circular trajectory. It is also worth pointing out that the curves of the proposed scheme and the HFH trajectory are very close to each other, while the latter can realize a little higher sum rate. Nevertheless, the HFH trajectory and circular trajectory only focus on maximizing the sum rate of UAV-served users without considering the BS-served users. In contrary, the interference from the UAV to the BS is restricted to a small threshold in our proposed scheme, which can guarantee the QoS of BS-served users at the cost of sacrificing the throughput of UAV-served users. Thus, our proposed scheme is most suitable to be utilized in this scenario.

In Fig. 4, the sum rate of BS-served users is compared for different values of $P_{t h}, M$ and $\gamma$, when Scheme I in Section IV-A is adopted. From the results, we can see that the sum rate of the BS-served users increases with $P_{t h}$, as higher transmit power of the BS will improve the transmission performance, with the interference to the UAV-served users 


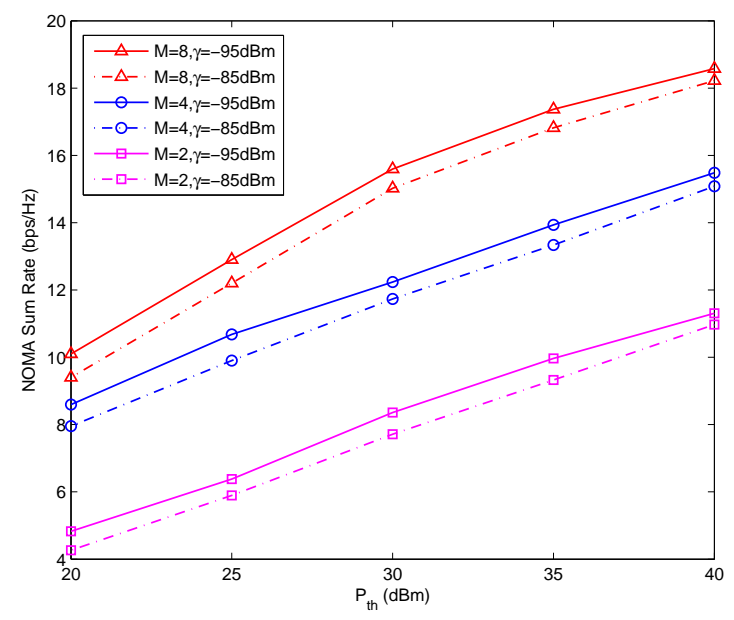

Fig. 4. Sum rate of BS-served users with different values of $P_{t h}, M$ and $\gamma$ in Scheme I. $K=3$ and $I=3$.

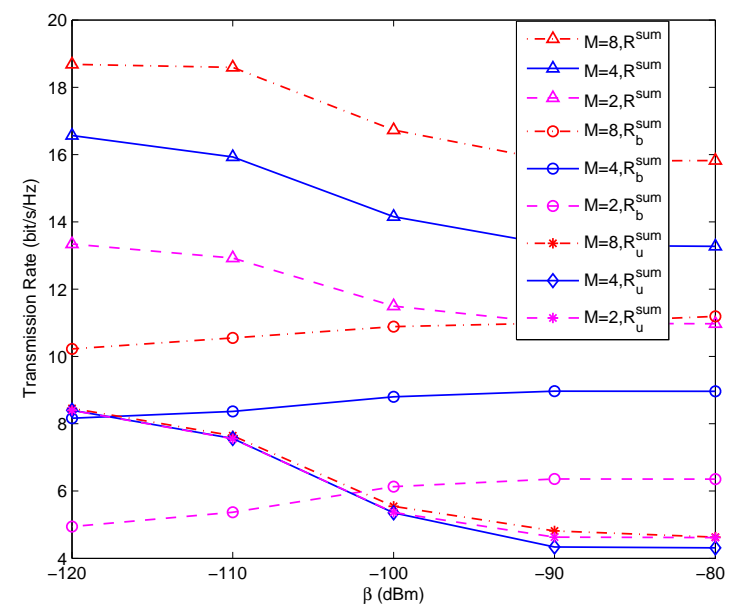

Fig. 5. Comparison of $R^{\text {sum }}, R_{b}^{\text {sum }}$ and $R_{u}^{\text {sum }}$ with different values of $M$ and $\beta$ in Scheme II. $K=3$ and $I=3$.

properly managed. In addition, we can see that when $M$ is larger, the sum rate will become higher. This is because more antenna resource can be utilized in the joint precoding to achieve better performance. Furthermore, the sum rate will increase with smaller value of $\gamma$, due to smaller interference from the UAV.

In Fig. 5, the $R^{\text {sum }}, R_{b}^{\text {sum }}$ and $R_{u}^{\text {sum }}$ are compared when Scheme II is adopted, for different values of $M$ and $\beta . P_{t h}=$ $20 \mathrm{dBm}$ and $\gamma=-90 \mathrm{dBm}$. From the results, we can see that the sum rate of the UAV-served users $R_{u}^{\text {sum }}$ becomes lower with larger $\beta$, as larger interference will be generated from the BS to the UAV-served user. On the other hand, the sum rate of the BS-served users $R_{b}^{\text {sum }}$ increases with $\beta$, because the constraint in $(42 \mathrm{c})$ can be relaxed. Thus, the sum rate of the whole cellular network $R^{\text {sum }}$ decreases with $\beta$, according to $R_{u}^{\text {sum }}$ and $R_{b}^{\text {sum }}$. Furthermore, $R_{b}^{\text {sum }}$ and $R^{\text {sum }}$ both increase with $M$, because larger $R_{b}^{s u m}$ can be achieved when more antennas are utilized in the beamforming. Nevertheless, $R_{u}^{\text {sum }}$ remains almost the same with different values of $M$, due to the fact that the performance of UAV-served users is only affected by $\beta$ from the BS, instead of $M$.

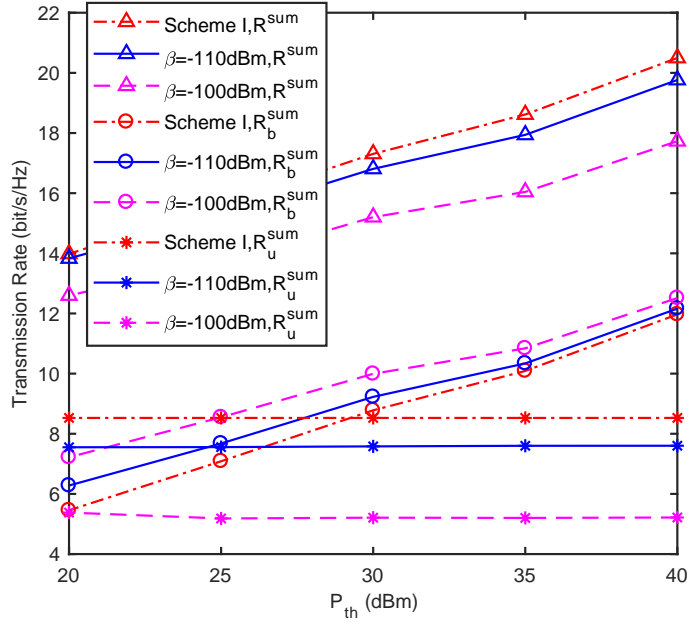

Fig. 6. Performance comparison of the proposed Scheme I and Scheme II when $M=2 . K=3$ and $I=3$.

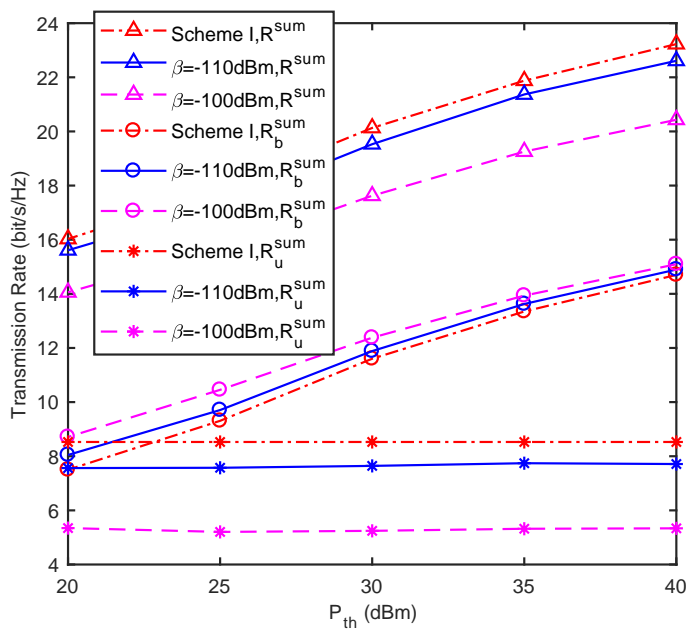

Fig. 7. Performance comparison of the proposed Scheme I and Scheme II when $M=3 . K=3$ and $I=3$.

In Figs. 6 and 7, the performances of the proposed Scheme I and Scheme II are compared for $M=2$ and $M=3$, respectively. Here, $\gamma=-90 \mathrm{dBm}$ and $p=20 \mathrm{~mW}$. From the results, we can see that the sum rate of the UAV-served users $R_{u}^{\text {sum }}$ and the sum rate of the total cellular network $R^{\text {sum }}$ in Scheme I are both higher than those in Scheme II, due to the fact that the interference from the BS is zero-forced at the UAV-served user. On the other hand, the sum rate of the BSserved users $R_{b}^{\text {sum }}$ in Scheme II is higher than that in Scheme I, because the relaxed constraint (42c) is considered, instead of perfect zero-forcing. In addition, $R_{b}^{\text {sum }}$ and $R^{\text {sum }}$ in these two schemes both increase with $P_{t h}$; however, $R_{u}^{\text {sum }}$ remains unchanged with different values of $P_{t h}$. This is because the power of the interference from the BS to the UAV-served user is either zero-forced or limited by $\beta$. Furthermore, we can also see that $R_{u}^{\text {sum }}$ decreases with $\beta$ while $R_{b}^{\text {sum }}$ increases with $\beta$, due to the fact that stricter interference constraint in (42c) means worse $R_{b}^{\text {sum }}$ but better $R_{u}^{\text {sum }}$. Last, comparing Figs. 6 and 7 , we can observe that when more antennas are equipped at the BS, i.e., larger value of $M, R_{b}^{\text {sum }}$ increases but $R_{u}^{\text {sum }}$ remains almost unchanged. Thus, we can adopt Scheme II to 


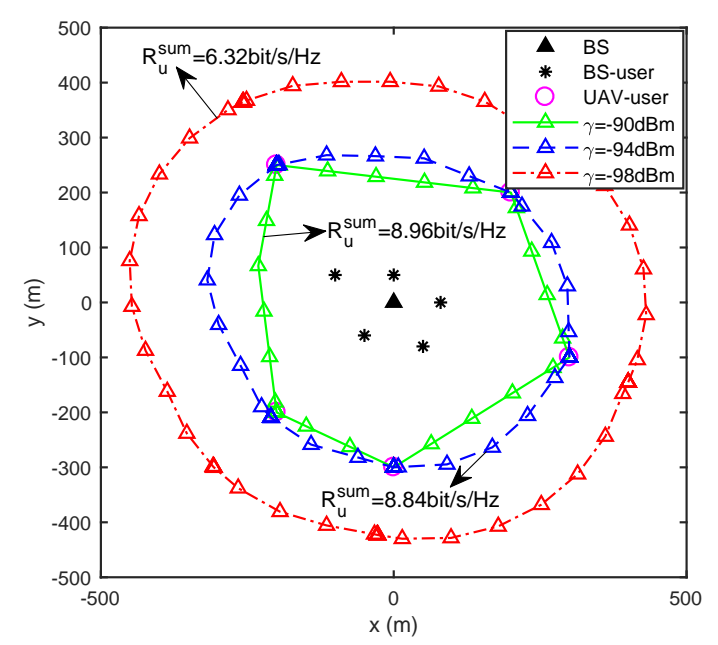

Fig. 8. Optimal UAV trajectories with different values of $\gamma . K=5$ and $I=5$. The UAV location of each time slot is marked by ' $\triangle$ '.

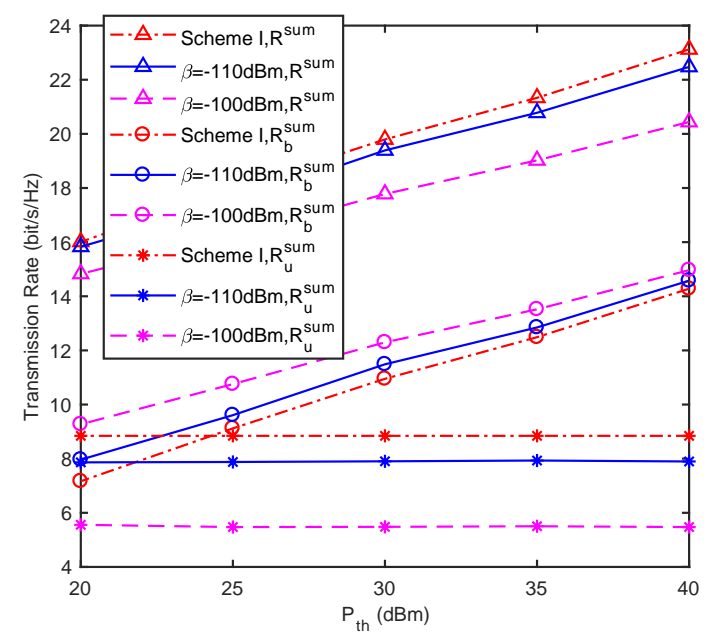

Fig. 9. Performance comparison of the proposed Scheme I and Scheme II when $M=2 . K=5$ and $I=5$.

improve the performance of the BS-served users if needed, when the antennas at the BS are inadequate.

Then, to further investigate the performance of the proposed scheme, we consider more users in the cellular network, i.e., $K=5 \mathrm{BS}$-served users and $I=5 \mathrm{UAV}$-served users according to the topology in Fig. 8. The optimal UAV trajectories via Algorithm 1 are shown with different values of $\gamma$ in Fig. 8 . In the simulation, $\mathcal{N}=60, v=50 \mathrm{~m} / \mathrm{s}, \sigma^{2}=-110 \mathrm{dBm}$, $\rho_{u}=-60 \mathrm{~dB}, \rho_{b}=-40 \mathrm{~dB}, \alpha=3, \mathcal{T}=100 \mathrm{~s}, p=20 \mathrm{~mW}$, $P_{t h}=20 \mathrm{dBm}$, and Scheme I of the NOMA precoding are considered. From the results, we can observe that a pentagon trajectory can be approximately achieved via connecting these five UAV-served users, in the case of $\gamma=-90 \mathrm{dBm}$ with $R_{u}^{s u m}=8.96 \mathrm{bit} / \mathrm{s} / \mathrm{Hz}$. This is because larger $\gamma$ means higher power of interference that can be generated to the BS-served users, which can relax the constraint (18c) and lead to better performance of UAV-served users. On the other hand, the interference constraint will be much stricter with smaller $\gamma$, and the UAV should fly away from the BS-served users with lower $R_{u}^{\text {sum }}$ to avoid interference.

In Figs. 9 and 10, the performance of the proposed Scheme

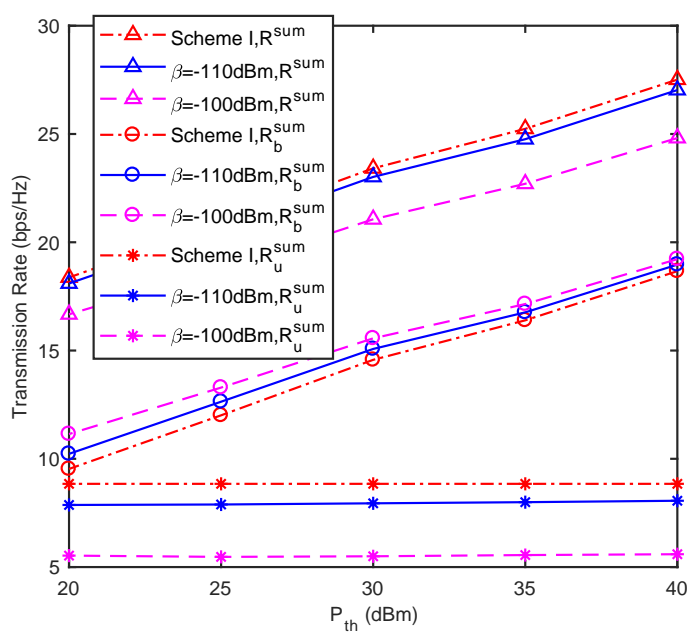

Fig. 10. Performance comparison of the proposed Scheme I and Scheme II when $M=3 . K=5$ and $I=5$.

I and Scheme II is compared with $M=2$ and $M=3$, respectively, with more users involved, i.e., $K=5$ and $I=5$. $\gamma=-94 \mathrm{dBm}$ and $p=20 \mathrm{~mW}$. From the results, we can observe that $R^{\text {sum }}$ and $R_{u}^{\text {sum }}$ in Scheme I are both higher than those in Scheme II, due to the fact that the interference from the BS can be zero-forced at the UAV-served user in each time slot via Scheme I. On the other hand, the relaxed interference from the BS to the UAV-served user according to (42c) in Scheme II will increase $R_{b}^{\text {sum }}$ with lower $R_{u}^{\text {sum }}$. In addition, $R_{b}^{\text {sum }}$ and $R^{\text {sum }}$ in these two schemes both increase with $P_{t h}$, with $R_{u}^{\text {sum }}$ almost unchanged. This is because the interference from the BS to the UAV-serve user in each time slot of these two schemes is either zero-forced or constrained by $\beta$ in (42c). Furthermore, we can also notice that larger $\beta$ will lead to higher $R_{b}^{\text {sum }}$ but lower $R_{u}^{\text {sum }}$, due to the relaxed constraint (42c). Last, comparing Figs. 9 and 10, we can see that more antennas at the BS will result in higher $R_{b}^{\text {sum }}$, but with $R_{u}^{\text {sum }}$ almost unchanged. Thus, Scheme II can be adopted to further improve the performance of BS-served users comparing to Scheme I, when the number of antennas at the BS is limited.

\section{CONCLUSIONS}

In this paper, the trajectory of UAV and the precoding vectors of NOMA BS were jointly optimized to maximize the sum rate for UAV-assisted NOMA networks, in which the users are served by the BS or UAV separately. This joint optimization problem is extremely difficult to solve, and thus we divided it into two subproblems. First, the UAV trajectory and transmission scheduling were optimized to maximize the sum rate of UAV-served users, with the interference to the BSserved users constrained. Then, two schemes were proposed to optimize the precoding vectors of NOMA BS, with its interference to the UAV-served user zero-forced or limited, respectively. Due to the non-convexity of the above problems, effective sub-optimal solutions were proposed to solve them with lower computational complexity. Simulation results were finally presented to show the effectiveness and efficiency of the proposed schemes. It can be concluded that the UAV should 
fly close to its served users while staying away from the BSserved users to guarantee the performance.In our future work, some fundamental issues of energy consumption and backhaul will be considered [42], and more practical UAV-to-ground channel models will be investigated [43].

\section{REFERENCES}

[1] X. Pang, Z. Li, X. Chen, Y. Cao, N. Zhao, Y. Chen, and Z. Ding, "UAVaided NOMA networks with optimization of trajectory and precoding," in Proc. WCSP'18, pp. 1-6, Hangzhou, China, Oct. 2018.

[2] Z. Li, L. Guan, C. Li, and A. Radwan, "A secure intelligent spectrum control strategy for future $\mathrm{THz}$ mobile heterogeneous networks," IEEE Commun. Mag., vol. 56, no. 6, pp. 116-123, Jun. 2018.

[3] Z. Ding, X. Lei, G. K. Karagiannidis, R. Schober, J. Yuan, and V. K. Bhargava, "A survey on non-orthogonal multiple access for $5 \mathrm{G}$ networks: Research challenges and future trends," IEEE J. Sel. Areas Commun., vol. 35, no. 10, pp. 2181-2195, Oct. 2017.

[4] Z. Ding, Y. Liu, J. Choi, Q. Sun, M. Elkashlan, C.-L. I, and H. V. Poor, "Application of non-orthogonal multiple access in LTE and 5G networks," IEEE Commun. Mag., vol. 55, no. 2, pp. 185-191, Feb. 2017.

[5] L. Dai, B. Wang, Y. Yuan, S. Han, C.-L. I, and Z. Wang, "Nonorthogonal multiple access for 5G: solutions, challenges, opportunities, and future research trends," IEEE Commun Mag., vol. 53, no. 9, pp. 7481, Sep. 2015

[6] Q. Wu, W. Chen, D. W. K. Ng, and R. Schober, "Spectral and energyefficient wireless powered IoT networks: NOMA or TDMA?," IEEE Trans. Veh. Technol., vol. 67, no. 7, pp. 6663-6667, Jul. 2018.

[7] S. Wei, J. Li, W. Chen, H. Su, Z. Lin, and B. Vucetic, "Power adaptive network coding for a non-orthogonal multiple-access relay channel," IEEE Trans. Commun., vol. 62, no. 3, pp. 872-887, Mar. 2014.

[8] P. Wang, J. Xiao, and L. Ping, "Comparison of orthogonal and nonorthogonal approaches to future wireless cellular systems," IEEE Veh. Technol. Mag., vol. 1, no. 3, pp. 4-11, Sep. 2006.

[9] Z. Chen, Z. Ding, X. Dai, and R. Zhang, "An optimization perspective of the superiority of NOMA compared to conventional OMA," IEEE Trans. Signal Process., vol. 65, no. 19, pp. 5191-5202, Oct. 2017.

[10] Z. Yang, Z. Ding, P. Fan, and N. Al-Dhahir, "A general power allocation scheme to guarantee quality of service in downlink and uplink NOMA systems," IEEE Trans. Wireless Commun., vol. 15, no. 11, pp. 7244 7257, Nov. 2016.

[11] Z. Ding, P. Fan, and H. V. Poor, "Impact of user pairing on 5G nonorthogonal multiple-access downlink transmissions," IEEE Trans. Veh. Technol., vol. 65, no. 8, pp. 6010-6023, Aug. 2016.

[12] M. F. Hanif, Z. Ding, T. Ratnarajah, and G. K. Karagiannidis, "A minorization-maximization method for optimizing sum rate in the downlink of non-orthogonal multiple access systems," IEEE Trans. Signal Process., vol. 64, no. 1, pp. 76-88, Jan. 2016.

[13] Z. Ding, F. Adachi, and H. V. Poor, "The application of MIMO to nonorthogonal multiple access," IEEE Trans. Wireless Commun., vol. 15, no. 1, pp. 537-552, Jan. 2016.

[14] H. Lin, F. Gao, S. Jin, and G. Y. Li, "A new view of multi-user hybrid massive MIMO: Non-orthogonal angle division multiple access," IEEE J. Sel. Areas Commun., vol. 35, no. 10, pp. 2268-2280, Oct. 2017.

[15] B. Chen, Y. Chen, Y. Chen, Y. Cao, N. Zhao, and Z. Ding, "A novel spectrum sharing scheme assisted by secondary NOMA relay," IEEE Wireless Commun. Lett., vol. 7, no. 5, pp. 732-735, Oct. 2018.

[16] Y. Wu, L. P. Qian, H. Mao, X. Yang, H. Zhou, and X. S. Shen, "Optimal power allocation and scheduling for non-orthogonal multiple access relay-assisted networks," IEEE Trans. Mob. Comput., vol. 17, no. 11, pp. 2591-2606, Nov. 2018.

[17] Z. Zhao, M. Xu, Y. Li, and M. Peng, "A non-orthogonal multiple accessbased multicast scheme in wireless content caching networks," IEEE J. Sel. Areas Commun., vol. 35, no. 12, pp. 2723-2735, Dec. 2017.

[18] X. Zhu, C. Jiang, L. Kuang, N. Ge, and J. Lu, "Non-orthogonal multiple access based integrated terrestrial-satellite networks," IEEE J. Sel. Areas Commun., vol. 35, no. 10, pp. 2253-2267, Oct. 2017.

[19] Y. Liu, Z. Qin, M. Elkashlan, A. Nallanathan, and J. A. McCann, "Nonorthogonal multiple access in large-scale heterogeneous networks," IEEE J. Sel. Areas Commun., vol. 35, no. 12, pp. 2667-2680, Dec. 2017.

[20] Z. Zhang, Z. Ma, Y. Xiao, M. Xiao, G. K. Karagiannidis, and P. Fan, "Non-orthogonal multiple access for cooperative multicast millimeter wave wireless networks," IEEE J. Sel. Areas Commun., vol. 35, no. 8, pp. 1794-1808, Aug. 2017
[21] Z. Zhang, Z. Ma, M. Xiao, Z. Ding, and P. Fan, "Full-duplex device-todevice-aided cooperative nonorthogonal multiple access," IEEE Trans. Veh. Technol., vol. 66, no. 5, pp. 4467-4471, May 2017.

[22] Y. Zeng, R. Zhang, and T. J. Lim, "Wireless communications with unmanned aerial vehicles: opportunities and challenges," IEEE Commun. Mag., vol. 54, no. 5, pp. 36-42, May 2016.

[23] J. Lyu, Y. and R. Zhang, and T. J. Lim, "Placement optimization of UAVmounted mobile base stations," IEEE Commun. Lett., vol. 21, no. 3, pp. 604-607, Mar. 2017.

[24] Y. Zeng, R. Zhang, and T. J. Lim, "Throughput maximization for UAV-enabled mobile relaying systems," IEEE Trans. Commun., vol. 64 no. 12, pp. 4983-4996, Dec. 2016.

[25] Y. Chen, N. Zhao, Z. Ding, and M.-S. Alouini, "Multiple UAVs as relays: Multi-hop single link versus multiple dual-hop links," IEEE Trans. Wireless Commun., vol. 17, no. 9, pp. 6348-6359, Sept. 2018.

[26] Y. Li and L. Cai, "UAV-assisted dynamic coverage in a heterogeneous cellular system," IEEE Network, vol. 31, no. 4, pp. 56-61, Jul.2017.

[27] J. Zhao, F. Gao, Q. Wu, S. Jin, Y. Wu, and W. Jia, "Beam tracking for UAV mounted satcom on-the-move with massive antenna array," IEEE J. Sel. Areas Commun., vol. 36, no. 2, pp. 363-375, Feb. 2018.

[28] Q. Wu, Y. Zeng, and R. Zhang, "Joint trajectory and communication design for UAV-enabled multiple access," in Proc. IEEE Globecom'17, pp. 1-6, Singapore, Singapore, Dec. 2017.

[29] Q. Wu, Y. Zeng, and R. Zhang, "Joint trajectory and communication design for multi-UAV enabled wireless networks," IEEE Trans. Wireless Commun., vol. 17, no. 3, pp. 2109-2121, Jan. 2018.

[30] J. Lyu, Y. Zeng, and R. Zhang, "Spectrum sharing and cyclical multiple access in UAV-aided cellular offloading," in Proc. IEEE Globecom'17, pp. 1-6, Singapore, Singapore, Dec. 2017.

[31] F. Cheng, S. Zhang, Z. Li, Y. Chen, N. Zhao, R. Yu, and V. C. M. Leung, "UAV trajectory optimization for data offloading at the edge of multiple cells," IEEE Trans. Veh. Technol., vol. 67, no. 7, pp. 6732-6736, Jul. 2018.

[32] N. Zhao, F. Cheng, F. R. Yu, J. Tang, Y. Chen, G. Gui, and H. Sari, "Caching UAV assisted secure transmission in hyper-dense networks based on interference alignment," IEEE Trans. Commun., vol. 66, no. 5, pp. 2281-2294, May 2018.

[33] Y. Zeng and R. Zhang, "Energy-efficient UAV communication with trajectory optimization," IEEE Trans. Wireless Commun., vol. 16, no. 6, pp. 3747-3760, Jun. 2017.

[34] D. Yang, Q. Wu, Y. Zeng, and R. Zhang, "Energy trade-off in ground-toUAV communication via trajectory design," IEEE Trans. Veh. Technol., vol. 67 , no. 7, pp. 6721-6726, Jul. 2018.

[35] Q. Wu and R. Zhang, "Common throughput maximization in UAV enabled OFDMA systems with heterogeneous delay consideration," IEEE Trans. Commun., vol. 66, no. 12, pp. 6614-6627, Dec. 2018.

[36] Q. Wu, X. Jie, and R. Zhang, "Capacity characterization of UAV-enabled two-user broadcast channel," IEEE J. Sel. Areas Commun., vol. 36, no. 9, pp. 1955-1971, Sept. 2018.

[37] A. A. Nasir, H. D. Tuan, T. Q. Duong, and H. V. Poor, "UAVenabled communication using NOMA." [Online] Available: https: //arxiv.org/abs/1806.03604.

[38] Y. Li, P. Fan, and N. C. Beaulieu, "Cooperative downlink max-min energy-efficient precoding for multicell MIMO networks," IEEE Trans. Veh. Technol., vol. 65, no. 11, pp. 9425-9430, Nov. 2016.

[39] Y. Cao, N. Zhao, F. R. Yu, M. Jin, Y. Chen, J. Tang, and V. C. M. Leung, "Optimization or alignment: Secure primary transmission assisted by secondary networks," IEEE J. Sel. Areas Commun., vol. 36, no. 4, pp. 905-917, Apr. 2018.

[40] K. Kreutz-Delgado, "The complex gradient operator and the CRcalculus." [Online] Available: https: //arxiv.org/abs/0906.4835.

[41] M. S. Lobo, L. Vandenberghe, S. Boyd, and H. Lebret, "Applications of second-order cone programming," Linear Algebra Appl., vol. 248, no. 1-3, pp. 193-228, Nov. 1998.

[42] Q. Wu, L. Liu, and R. Zhang, "Fundamental tradeoffs in communication and trajectory design for UAV-enabled wireless network," IEEE Wireless Commun., to appear.

[43] A. A. Khuwaja, Y. Chen, N. Zhao, M.-S. Alouini, and P. Dobbins, "A survey of channel modeling for UAV communications," IEEE Commun. Surveys Tuts., vol. 20, no. 4, pp. 2804-2821, 4th Quart. 2018. 


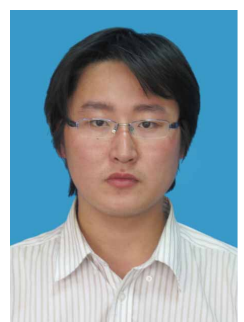

Nan Zhao (S'08-M'11-SM'16) is currently an Associate Professor at Dalian University of Technology, China. He received the Ph.D. degree in information and communication engineering in 2011, from Harbin Institute of Technology, Harbin, China.

Dr. Zhao is serving or served on the editorial boards of 7 SCI-indexed journals, including IEEE Transactions on Green Communications and Networking. He won the best paper awards in IEEE VTC 2017 Spring, MLICOM 2017, ICNC 2018, WCSP 2018 and CSPS 2018. He also received the IEEE Communications Society Asia Pacific Board Outstanding Young Researcher Award in 2018

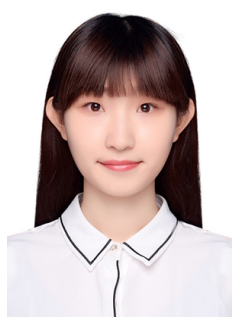

Xiaowei Pang received the B.S. degree in communication engineering from Northwestern Polytechnical University, Xi' an, China, in 2018. She is currently working toward the graduate degree in the School of Information and Communication Engineering, Dalian University of Technology, Dalian, China. Her current research interests include UAV communications, NOMA technique and optimization of wireless networks.

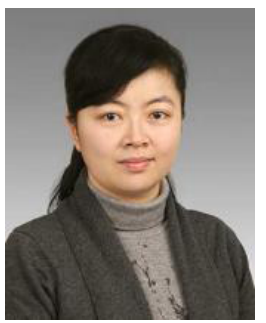

Zan Li (M'06-SM'14) received her B.S. degree in communications engineering and her M.S. and $\mathrm{Ph} . \mathrm{D}$. degrees in communication and information systems from Xidian University, Xian, China, in 1998, 2000, and 2004, respectively. She is currently a professor with the State Key Laboratory of Integrated Services Networks, School of Telecommunications Engineering, Xidian University. Her research interests include wireless communication and signal processing, particularly covert communication, weak signal detection, spectrum sensing, and cooperative

communication

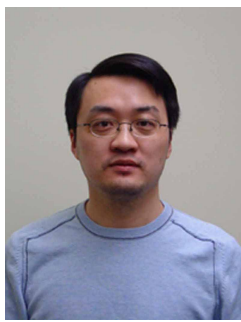

Yunfei Chen (S'02-M'06-SM'10) received his B.E. and M.E. degrees in electronics engineering from Shanghai Jiaotong University, Shanghai, P.R.China, in 1998 and 2001, respectively. He received his Ph.D. degree from the University of Alberta in 2006. $\mathrm{He}$ is currently working as an Associate Professor at the University of Warwick, U.K. His research interests include wireless communications, cognitive radios, wireless relaying and energy harvesting.

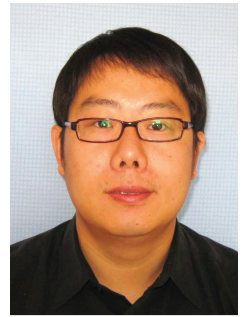

Feng $\mathbf{L i}$ received the B.S. and the M.S. degree from the Harbin University of Science and Technology, Harbin, China in 2001 and 2005, respectively. He also received his Ph.D degree from the Harbin Institute of Technology, Harbin, China in 2013. $\mathrm{He}$ is currently working at College of Information Engineering, Zhejiang University of Technology.

From 2005 to 2009 , he was with the Qiaohang communication company, Harbin, China, where he worked on the research and development of the digital trunking system. His research interests include cognitive radio networks, sensor networks and satellite systems.

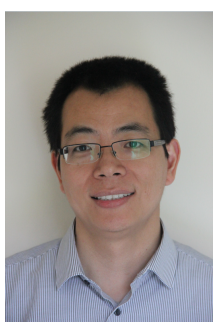

University.

Dr Ding' research interests are 5G networks, game theory, cooperative and energy harvesting networks and statistical signal processing. He is serving as an Editor for IEEE Transactions on Communications, IEEE Transactions on Vehicular Technology, and Journal of Wireless Communications and Mobile Computing, and was an Editor for IEEE Wireless Communication Letters, IEEE Communication Letters from 2013 to 2016. He received the best paper award in IET ICWMC-2009 and IEEE WCSP-2014, the EU Marie Curie Fellowship 2012-2014, the Top IEEE TVT Editor 2017, IEEE Heinrich Hertz Award 2018 and the IEEE Jack Neubauer Memorial Award 2018.

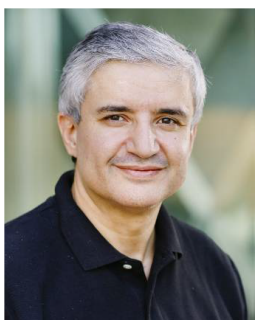

Mohamed-Slim Alouini (S'94-M'98-SM'03-F'09) was born in Tunis, Tunisia. He received the Ph.D. degree in Electrical Engineering from the California Institute of Technology (Caltech), Pasadena, CA, USA, in 1998. He served as a faculty member in the University of Minnesota, Minneapolis, MN, USA, then in the Texas A\&M University at Qatar, Education City, Doha, Qatar before joining King Abdullah University of Science and Technology (KAUST), Thuwal, Makkah Province, Saudi Arabia as a Professor of Electrical Engineering in 2009. His current research interests include the modeling, design, and performance analysis of wireless communication systems. 